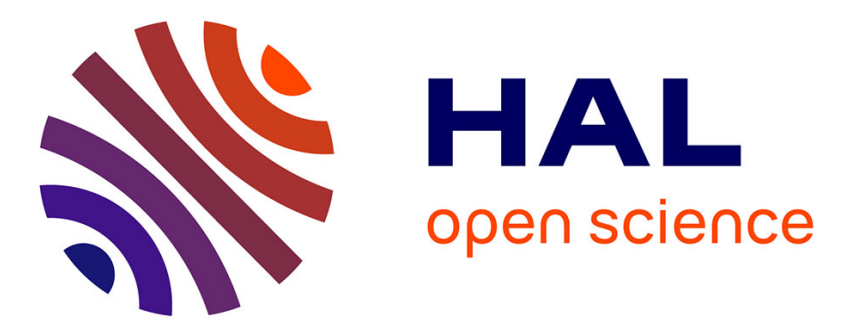

\title{
Asymptotic and transient behaviour for a nonlocal problem arising in population genetics
}

\author{
Jean-Baptiste Burie, R Djidjou-Demasse, Arnaud Ducrot
}

\section{To cite this version:}

Jean-Baptiste Burie, R Djidjou-Demasse, Arnaud Ducrot. Asymptotic and transient behaviour for a nonlocal problem arising in population genetics. European Journal of Applied Mathematics, 2020, 31 (1), pp.84-110. 10.1017/S0956792518000487 . hal-01874125

\section{HAL Id: hal-01874125 \\ https://hal.science/hal-01874125}

Submitted on 14 Sep 2018

HAL is a multi-disciplinary open access archive for the deposit and dissemination of scientific research documents, whether they are published or not. The documents may come from teaching and research institutions in France or abroad, or from public or private research centers.
L'archive ouverte pluridisciplinaire HAL, est destinée au dépôt et à la diffusion de documents scientifiques de niveau recherche, publiés ou non, émanant des établissements d'enseignement et de recherche français ou étrangers, des laboratoires publics ou privés. 


\title{
Asymptotic and transient behaviour for a nonlocal problem arising in population genetics
}

\author{
J.-B. Burie ${ }^{a}$, R. Djidjou-Demasse ${ }^{b}$ And A. Ducrot ${ }^{a}$ \\ ${ }^{a}$ Univ. Bordeaux, IMB, UMR 5251, F-33400 Talence, France \\ CNRS, IMB, UMR 5251, F-33400 Talence, France. \\ email: jean-baptiste.burie@u-bordeaux.fr, arnaud.ducrot@u-bordeaux.fr \\ ${ }^{b}$ UMR 1065, INRA, Villenave d'Ornon F-33882, France \\ email:ddramses@gmail.com
}

May 3, 2018

\begin{abstract}
This work is devoted to the study of an integro-differential system of equations modelling the genetic adaptation of a pathogen by taking into account both mutation and selection processes. First we study the asymptotic behaviour of the system and prove that it eventually converges to a stationary state. Next we more closely investigate the behaviour of the system in the presence of multiple evolutionary attractors. Under suitable assumptions and based on a small mutation variance asymptotic, we describe the existence of a long transient regime during which the pathogen population remains far from its asymptotic behaviour and highly concentrated around some phenotypic value that is different from the one described by its asymptotic behaviour. In that setting, the time needed for the system to reach its large time configuration is very long and multiple evolutionary attractors may act as a barrier of evolution that can be very long to bypass.
\end{abstract}

Key words Nonlocal equation, asymptotic behaviour, transient behaviour, population genetics.

2000 Mathematical Subject Classification 35B40, 35R09, 35Q92, 92D10. 


\section{Introduction}

The model. In this note we investigate the behaviour of the following nonlocal system of equations

$$
\left\{\begin{array}{l}
\frac{\mathrm{d} U(t)}{\mathrm{d} t}=\Lambda-\mu_{u} U(t)-U(t) \int_{\mathbb{R}^{N}} \beta(y) L[v(t, .)](y) \mathrm{d} y, t>0, \\
\frac{\partial v(t, x)}{\partial t}=\beta(x) U(t) L[v(t, .)](x)-\mu_{v} v(t, x), t>0, x \in \mathbb{R}^{N},
\end{array}\right.
$$

wherein $L$ denotes the integral operator

$$
L[v(t, .)](x)=\int_{\mathbb{R}^{N}} J(x-y) r(y) v(t, y) \mathrm{d} y .
$$

This model was proposed by Iacono et al. in [13] in a slightly more complex setting with a focus on plant epidemiology and pathogen adaptation. The authors studied in particular the impact of the introduction of resistant plants on the plant-pathogen system using various indicators. Moreover this model follows the population genetics approach (rather than the classical adaptive dynamics one) advocated in [7]. In this approach, epidemiological and evolution processes may occur on the same time scale, a mutation kernel is introduced, and multiple pathogen strains are considered simultaneously. However here, as in e.g. [3], these multiple strains will be described with a continuous variable rather than a discrete one.

More precisely in the system (1.1), $t \geq 0$ denotes the time and $x \in \mathbb{R}^{N}$ denotes a phenotypic trait value and can be treated as a "label" of the genotype (or strain) of the pathogen. The phenotypic trait value $x$ is assumed to be possibly multi-dimensional, namely $N \geq 1$ is a fixed integer. The state variables $U=U(t)$ and $v=v(t, x)$ denote the density of healthy and infected plants at time $t$ with a pathogen phenotype $x$ respectively. Healthy plants renew with a constant influx $\Lambda>0$ and exit with the natural death rate $\mu_{u}>0$. Infected plants exit at rate $\mu_{v}>0$. Here Model (1.1)-(1.2) takes into account a continuum of different pathogen strains indexed by $x \in \mathbb{R}^{N}$. This modelling approach allows to address the issue of pathogen adaptation to quantitative resistance for example: all the pathogen strains cause infection but each with its own level of quantitative pathogenicity [15]. In that context, each pathogen strain is characterized by its phenotypic value $x$ which affects the life-history traits of the pathogens expressed during the host-pathogen interaction: infection efficiency $\beta(x)$ and production rate $r(x)$. The evolution in the space of phenotypic values is modelled by an integral operator with kernel $J(x-y)$ describing mutations from a pathogen strain with phenotypic value $y \in \mathbb{R}^{N}$ to another one with phenotypic value $x \in \mathbb{R}^{N}$.

Some properties of System (1.1)-(1.2) (written in a more general form) have been investigated by Djidjou-Demasse et al. in [9]. The authors have studied the existence and uniqueness of an endemic equilibrium. The profile of this endemic equilibrium point in the space of phenotypic values has been described 
when the mutation kernel $J$ depends on a small positive parameter $\varepsilon \ll 1$ and is highly concentrated with scaling form

$$
J_{\varepsilon}(x)=\frac{1}{\varepsilon^{N}} J\left(\frac{x}{\varepsilon}\right) .
$$

The analysis provided in the aforementioned work relies on the properties of the so-called fitness function $\Psi$ defined, in the setting of Problem (1.1)-(1.2), by

$$
\Psi(x)=\beta(x) r(x),
$$

that allows to take into account evolutionary interactions between efficiency and production rate of the pathogens. Here when $\varepsilon>0$ is very small then, under reasonable and rather generic assumptions, the endemic equilibrium concentrates on the set $\mathcal{S}$ of phenotypic values where the fitness function reaches its global maximum. Following the classical adaptive dynamics theory [8, 12, 17], this set $\mathcal{S}$ is referred to as the set of Evolutionary Attractors (EAs for short). Though several EAs may exist, in rather general situations the endemic equilibrium concentrates on a single "strongest" phenotypic value $\widehat{x} \in \mathcal{S}$ maximizing the fitness function. Thus in [9] the authors defined a suitable order relation on the EAs-set that allows to identify this "strongest" phenotypic value (or pathogen strain) when the fitness function $\Psi$ has at least two global maximum points. It roughly corresponds to the maximum with the flattest fitness shape.

Concentration properties of steady state solutions for nonlocal mutation selection models of ecological problems with respect to a continuous phenotypic trait have already been investigated, see for instance $[2,3,4,6]$ and references cited therein. In these works, the existence of stationary solutions is obtained using the spectral properties of some linear operators (infinite dimensional version of Perron-Frobenius Theorem).

For our model, as in [6], only a single component of the population is likely to mutate, and the existence of stationary solutions is directly related to the spectral properties of some linear operator using again the Perron-Frobenius like Theorem. In [4] such a spectral argument is coupled with a fixed point argument to study the properties of steady states while [3] studies the large time asymptotic of some nonlinear problem with concentration effect for small mutation parameter. Moreover, for a nonlocal mutation selection model, a time asymptotic convergence towards steady state solutions with concentration properties has been studied in [2].

In this work we shall study some dynamical properties of Problem (1.1)-(1.2). We shall first focus on the asymptotic behaviour $t \rightarrow \infty$ of the model. Then we shall describe some situations where this asymptotic behaviour is reached after a long transition period. Unlike the classical adaptive dynamics theory that focuses on the asymptotic behaviour of the evolutionary problem, the population genetics modelling approach allows to take into account the transient dynamics of the solutions. This analysis of the transient behaviour will be performed using the scaling form (1.3) for the mutation kernel with small $\varepsilon$. 
Applications and numerical simulations. A practical application we have in mind concerns disease control in agriculture. Indeed, it is now possible to devise disease resistant varieties. The development of a resistant variety is usually achieved within 5 to 10 years, while the resistance effectiveness turns out to be generally bypassed within 5 years [23]. A natural and important question is to understand how to extend and maximize the durability of the resistant variety. The purpose of the analysis performed in this note is to shed some light on how using multiple EAs as an evolution barrier can prevent the pathogen from adaptation during a very long period of time.

To further motivate our analysis, we consider two host populations (1) and (2) associated with pathogen fitness function $\Psi_{1}(x)$ and $\Psi_{2}(x)$ respectively. When well mixed with proportion $\chi \in(0,1)$ of the first population and $(1-\chi)$ for the second host population, the global pathogen fitness of the environment corresponds to

$$
\Psi(x)=\chi \Psi_{1}(x)+(1-\chi) \Psi_{2}(x), \forall x \in \mathbb{R}^{N} .
$$

Typically the functions $\Psi_{1}$ and $\Psi_{2}$ exhibit a single peak, meaning that the adaptation of the pathogen on the host populations (1) and (2) corresponds to a single phenotype value which may differ for each host. Therefore the global landscape fitness typically displays two peaks (adaptive fitness landscape) with possibly different levels, depending on the proportion $\chi$.

Let us display some numerical simulations of the one-dimensional model (1.1)-(1.2). In order to illustrate different possible configurations for the fitness function, $\Psi$, and their consequences on the dynamical behaviour for the solutions of (1.1)-(1.2) we choose

$$
\begin{aligned}
& \Psi_{1}(x)=\left\{\begin{array}{l}
-\frac{1}{2} \sin (a(x-2)) \text { if } x \in\left[2-\frac{\pi}{a}, 2\right], \\
0 \text { else, }
\end{array}\right. \\
& \Psi_{2}(x)=\left\{\begin{array}{l}
2(x-2)(3-x) \text { if } x \in[2,3] \\
0 \text { else }
\end{array}\right.
\end{aligned}
$$

wherein $a>0$ is a parameter.

When $\chi \neq \frac{1}{2}$ then the fitness function $\Psi$ defined in (1.4) has a single global maximum achieved on a unique EA, see Figure 1. In that case the solution of (1.1)-(1.2) rapidly stabilizes on the unique endemic stationary state that is highly concentrated, when $\varepsilon \ll 1$, on the unique EA.

When $\chi=\frac{1}{2}$, function $\Psi$ has two global maxima at $x_{1}=2-\frac{\pi}{2 a}$ and $x_{2}=\frac{5}{2}$. In that particular case, the dynamical behaviour of the solutions of (1.1)-(1.2) is much more complicated involving a possibly long transient behaviour.

Figure 2 corresponds to the generic case when $\Psi^{\prime \prime}\left(x_{1}\right) \neq \Psi^{\prime \prime}\left(x_{2}\right)$ with $a=2$. The initial pathogen population is supported around $x_{2}$. Then the solution exhibits first a transient concentration of the pathogen population around $x_{2}$ during a long time interval. After this transient stage, the pathogen population shifts and concentrates around the 'stronger' phenotypic value $x_{1}$, the flattest 

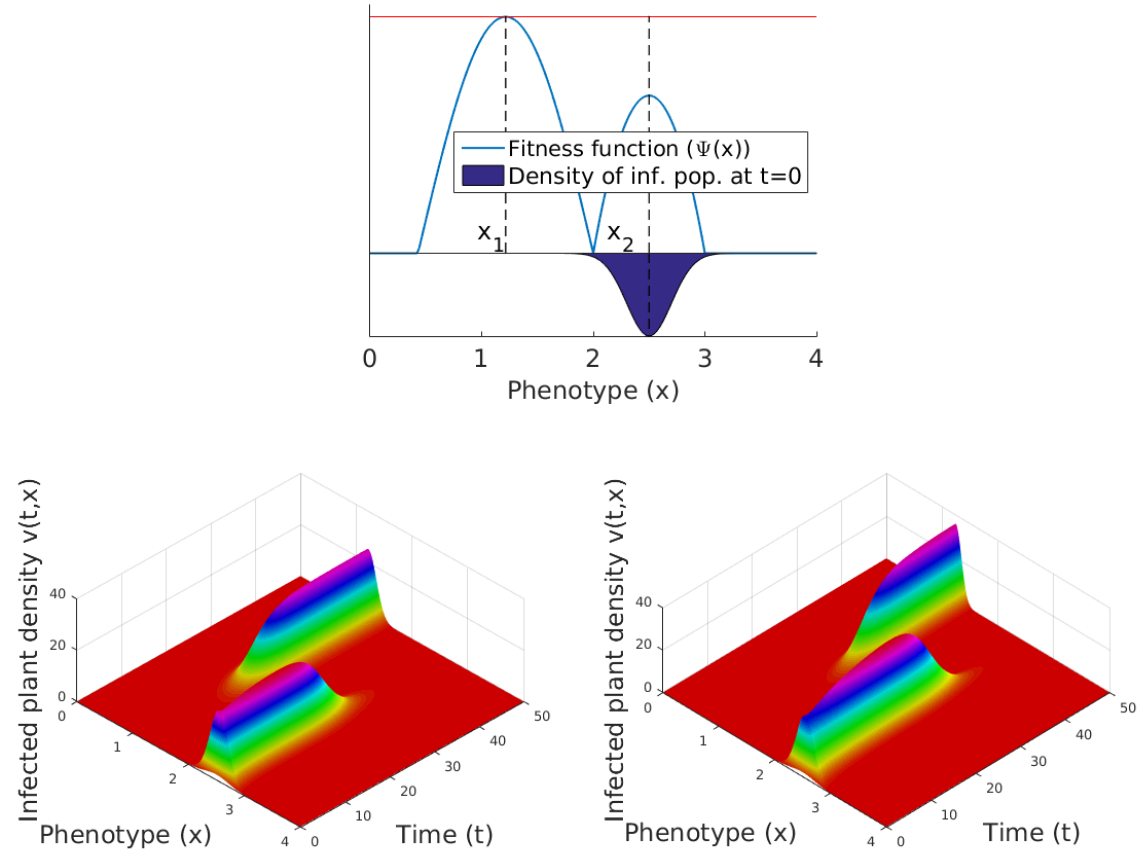

Figure 1: Fast convergence of the solutions of System (1.1)-(1.2) towards the asymptotic configuration. Parameter values are: $\chi=0.6$ and $a=2$ (fitness function), $\Lambda=10, \mu=1, \mu_{v}=1.05, \beta \equiv 1, J$ is the standard normal distribution. Initial populations are $U_{0}=\Lambda / \mu$ and $v_{0}$ a Gaussian function. (Top) The fitness function $\Psi$ and the density of infected population at time $t=0$. (Left) Time evolution of the infected population with $\varepsilon=0.04$. (Right) Time evolution of the infected population with $\varepsilon=0.02$. 
peak, for which $\Psi^{\prime \prime}\left(x_{1}\right)<\Psi^{\prime \prime}\left(x_{2}\right)$. Dividing $\varepsilon$ by two roughly multiply the duration of the transient stage by two. From numerical simulations, the duration of the transient stage is somehow proportional to $\frac{1}{\varepsilon}$ when $\varepsilon \ll 1$.
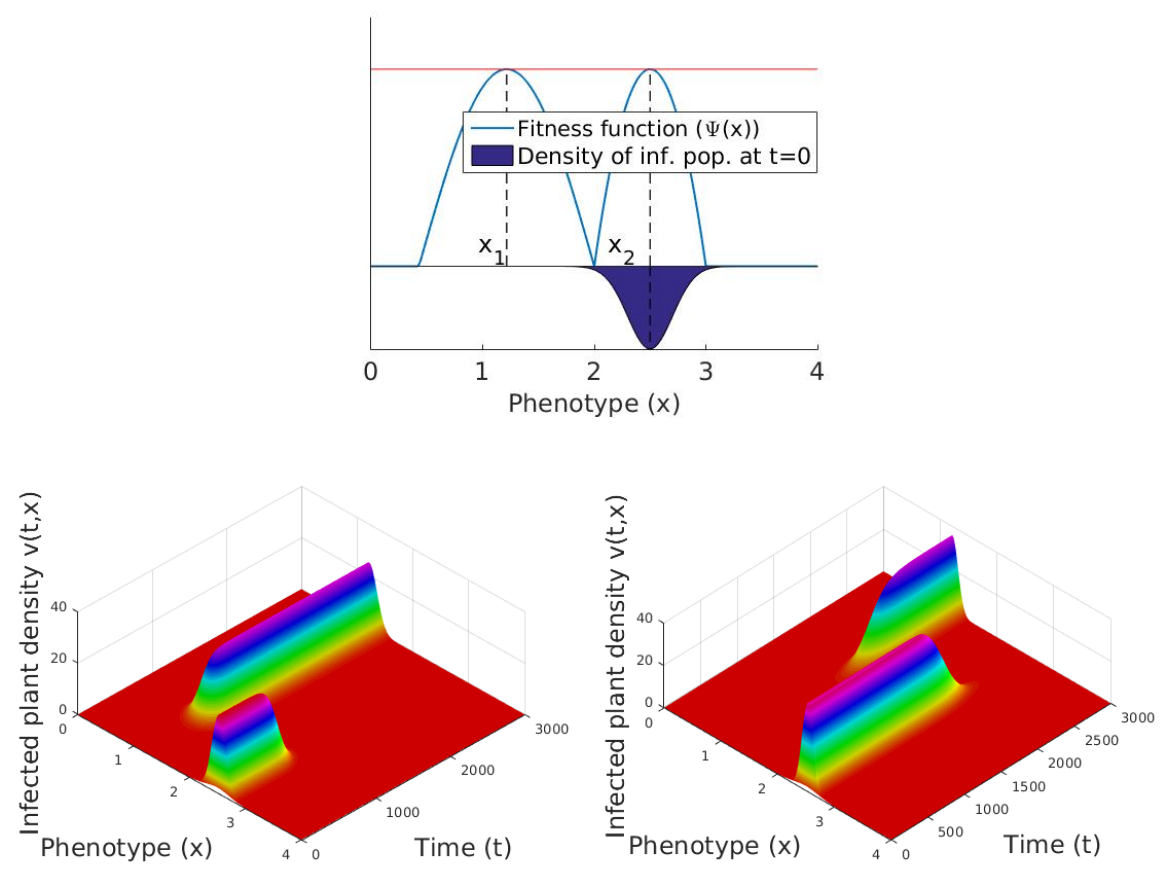

Figure 2: Slow dynamics behaviour of System (1.1)-(1.2) towards the asymptotic configuration. Parameter values are as in Figure 1 except $\chi=1 / 2$. (Top) The fitness function $\Psi$ and the density of infected population at time $t=0$. (Left) Time evolution of the infected population with $\varepsilon=0.04$. (Right) Time evolution of the infected population with $\varepsilon=0.02$.

Finally, Figure 3 corresponds to the case $a=2 \sqrt{2}$ when the derivatives $\Psi^{(n)}\left(x_{1}\right)$ and $\Psi^{(n)}\left(x_{2}\right)$ do not differ for $n=0,1,2,3$ and $\Psi^{(4)}\left(x_{1}\right)>\Psi^{(4)}\left(x_{2}\right)=$ 0. Again, after some long transient stage, the pathogen will shift from the phenotypic value $x_{1}$ to value $x_{2}$, with an increased transient time as $\varepsilon$ decreases. Here dividing $\varepsilon$ by 2 roughly multiplies the duration of the transient regime by 8. For this situation, $x_{1}$ again corresponds to the 'stronger' phenotypic value according to the results in [9]. Note that $x_{1}$ corresponds to the flatter peak since $\Psi\left(x_{1}+h\right)=\Psi\left(x_{2}+h\right)+\frac{1}{4 !} \Psi^{(4)}\left(x_{1}\right) h^{4}+o\left(h^{4}\right)$ when $h \rightarrow 0$ with $\Psi^{(4)}\left(x_{1}\right)>0$. From numerical simulations, in that particular situation and in contrast to the situation presented in Figure 2, the duration of the transient regime seems to depend nonlinearly upon $\varepsilon$ as $\frac{1}{\varepsilon^{3}}$ when $\varepsilon \ll 1$. 

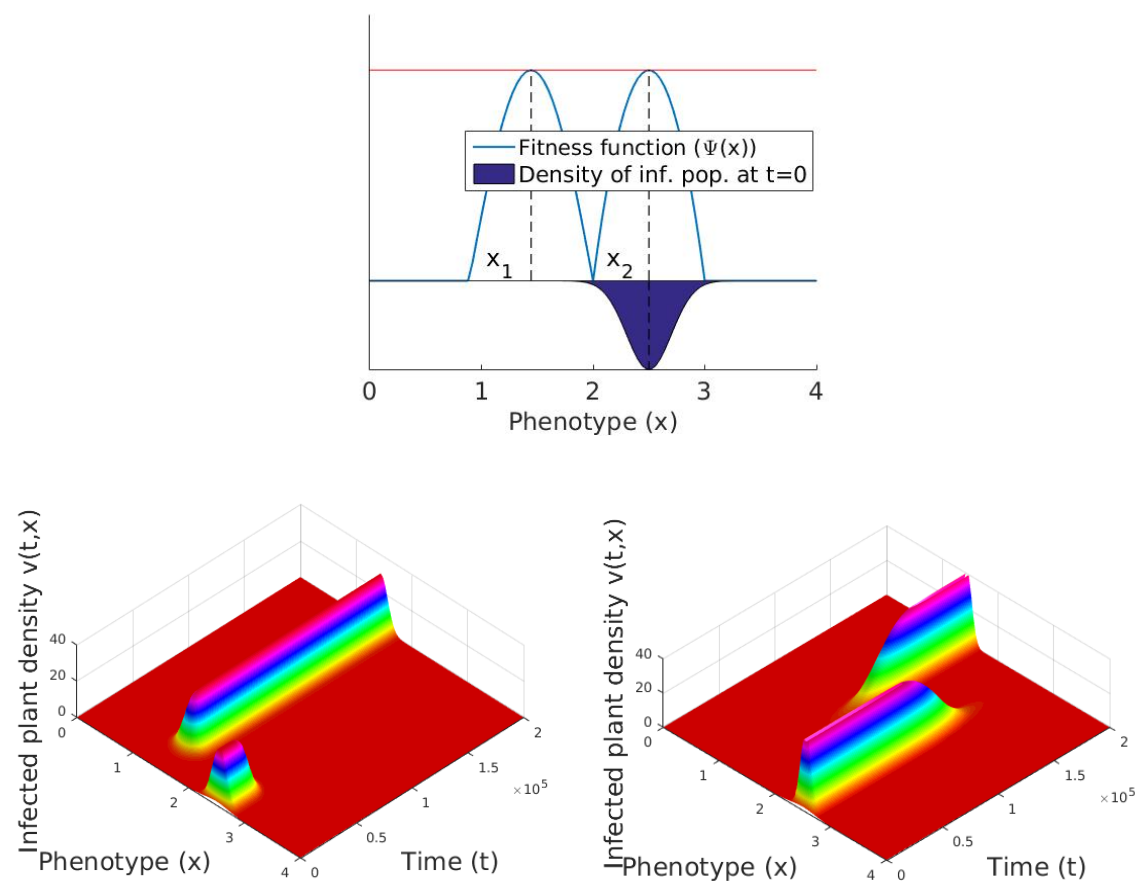

Figure 3: Slow dynamics behaviour of System (1.1)-(1.2) towards the asymptotic configuration. Parameter values are as in Figure 1 except $\chi=1 / 2$. (Top) The fitness function $\Psi$ and the density of infected population at time $t=0$. (Left) Time evolution of the infected population with $\varepsilon=0.04$. (Right) Time evolution of the infected population with $\varepsilon=0.02$. 
Aims. In this article we shall study the situation illustrated by Figure 3 in which the transient regime is very long as $\varepsilon$ becomes small and where the EA $x_{2}$ acts as an evolution barrier during this long transient period. The precise mathematical justification of such a behaviour involving slow motion regime around $x_{2}$ when $\varepsilon \ll 1$ is our main objective. We shall also focus on estimating the duration of this transient regime as a function of $\varepsilon \ll 1$. Such a phenomenon is usually referred to as a metastable dynamics whose understanding has attracted a lot of interest. Among others, let us mention the slow motion shock layer for viscous conservation laws $[14,20,21,16]$ and phase transition problems described by the Allen-Cahn equation for which we refer the readers to [5, 11]) and by the Cahn-Hilliard equation for which we refer to $[1,19]$.

The dynamical behaviour of System (1.1)-(1.2) is somehow reminiscent of the so-called 'survival of the flattest' phenomenon observed in quasi-species models of evolution: genotypes corresponding to flatter regions of the fitness function are more robust to high rates of mutations [22]. With an initial infected population concentrated around a narrower peak of the fitness function, $x_{2}$, the population will shift towards the flatter peak, $x_{1}$. The time needed to shift decreases as $\varepsilon$ increases. Indeed, larger values of $\varepsilon$ correspond to mutants exploring a larger portion of the phenotype space, which allows the population to cross the fitness barrier. This mechanism may explain the metastability observed in artificial and natural evolution [18]. Note however that, in contrast with quasi-species models, for our system the infected population cannot eventually concentrate around a strictly lower fitness peak even if this peak is broader (see $[9])$.

This work is organized as follows. In Section 2 we state and discuss the main results that will be obtained in this work: (i) the large time dynamics and (ii) the transient behaviour around a quasi-equilibrium. Section 3 is devoted to the proof of the first main result, the asymptotic behaviour of System (1.1)-(1.2). Section 4 completes the proof of the main results that deal with the transient behaviour of System (1.1)-(1.2).

\section{Main results}

In this section we shall state the main results that will be discussed in this note. Before going further we first reformulate Problem (1.1)-(1.2) to introduce a self-adjoint mutation operator. To that aim we consider the functions

$$
\tilde{v}(t, x):=\sqrt{\frac{r(x)}{\beta(x)}} v(t, x), \Theta(x)=\sqrt{r(x) \beta(x)}, \text { and } \tilde{\beta}(x):=\sqrt{\frac{\beta(x)}{r(x)}} .
$$

Hence omitting the tilde for notational simplicity and setting $\mu_{u}=1, \mu_{v}=\mu>$ 0, Problem (1.1)-(1.2) - with $J=J_{\varepsilon}$ - re-writes

$$
\left\{\begin{array}{l}
\frac{\mathrm{d} U(t)}{\mathrm{d} t}=\Lambda-U(t)-U(t) \int_{\mathbb{R}^{N}} \beta(x) \Theta(x) J_{\varepsilon} *(\Theta v(t, \cdot))(x) \mathrm{d} x, \\
\frac{\partial v}{\partial t}(t, x)=U(t) \Theta(x) J_{\varepsilon} *(\Theta v(t, \cdot))(x)-\mu v(t, x),
\end{array}\right.
$$


wherein the symbol $*$ denotes the convolution product on $\mathbb{R}^{N}$. Throughout this work, instead of $\Psi$, function $\Theta=\sqrt{\Psi}$ will be referred below as the fitness function (i.e. $\Theta(x)$ is the fitness of the pathogen strain $x$ ).

We first describe the large time behaviour of the above system of equations for suitable non-negative initial data $\left(U_{0}, v_{0}\right)$. To state our first main result, we shall make the following assumption.

Assumption 2.1 We assume that $\Lambda$ and $\mu$ are positive parameters and $\mu \geq 1$. The positive function $\beta$ is assumed to be bounded and to belong to $L^{2}\left(\mathbb{R}^{N}\right) \cap$ $\mathcal{C}^{0}\left(\mathbb{R}^{N}\right)$ while the function $\Theta$ is positive and continuous on $\mathbb{R}^{N}$ with $\Theta(x) \rightarrow 0$ as $|x| \rightarrow \infty$.

The mutation kernel $J \in L^{1}(\mathbb{R}) \cap L^{\infty}(\mathbb{R})$ satisfies

$$
\int_{\mathbb{R}} J(x) \mathrm{d} x=1, \quad J(x)>0 \text { and } J(-x)=J(x) \text { a.e. in } \mathbb{R}^{N} .
$$

Now fix $\varepsilon>0$ and consider the bounded linear operator $L=L_{\varepsilon}$ on $L^{p}\left(\mathbb{R}^{N}\right)$ for any $p \in[1, \infty)$ defined by

$$
L_{\varepsilon}[\varphi](x)=\int_{\mathbb{R}^{N}} \Theta(x) J_{\varepsilon}(x-y) \Theta(y) \varphi(y) \mathrm{d} y .
$$

Here $J_{\varepsilon}$ is defined in (1.3). Under assumption 2.1, for each $\varepsilon>0$ and $p \in[1, \infty)$, this bounded linear operator is positive, compact and irreducible on $L^{p}\left(\mathbb{R}^{N}\right)$ and it is a self-adjoint operator in $L^{2}\left(\mathbb{R}^{N}\right)$. As a consequence it admits a spectral decomposition with positive eigenvalues $\left\{\lambda_{n}^{\varepsilon}\right\}_{n \geq 1}$ such that

$$
\lambda_{1}^{\varepsilon}>\lambda_{2}^{\varepsilon} \geq \lambda_{3}^{\varepsilon} \geq \cdots \geq \lambda_{n}^{\varepsilon} \text { with } \lambda_{n}^{\varepsilon} \rightarrow 0 \text { as } n \rightarrow+\infty .
$$

The corresponding set of eigenvectors $\left\{\varphi_{n}^{\varepsilon}\right\}_{n \geq 1}$ forms a Hilbert basis of $L^{2}\left(\mathbb{R}^{N}\right)$ and the principal eigenvector $\varphi_{1}^{\varepsilon}$ is positive on $\mathbb{R}^{N}$. Here one may keep in mind that throughout this note, one has assumed that $\left\|\varphi_{n}^{\varepsilon}\right\|_{L^{2}\left(\mathbb{R}^{N}\right)}=1, \forall n \geq 1, \varepsilon>$ 0 . Moreover one may also notice that, since $J \in L^{1}\left(\mathbb{R}^{N}\right) \cap L^{\infty}\left(\mathbb{R}^{N}\right)$ and since $\Theta$ is bounded, one also has $\varphi_{n}^{\varepsilon} \in \cap_{p \in[1, \infty)} L^{p}\left(\mathbb{R}^{N}\right)$, for any $n \geq 1$ and $\varepsilon>0$.

Using the above assumption and the subsequent notations we are now able to discuss the large time behaviour of (2.5). This analysis is related to the threshold numbers $\mathcal{R}_{0, j}^{\varepsilon}$ defined by

$$
\mathcal{R}_{0, j}^{\varepsilon}=\frac{\Lambda}{\mu} \lambda_{j}^{\varepsilon}, \quad j=1,2 .
$$

In the sequel, we set $\mathcal{R}_{0}^{\varepsilon}=\mathcal{R}_{0,1}^{\varepsilon}$ (the dominant threshold value). Let us also introduce the following notations

$$
U_{j}^{\varepsilon}=\frac{\mu}{\lambda_{j}^{\varepsilon}} \text { and } V_{j}^{\varepsilon}=\frac{\mathcal{R}_{0, j}^{\varepsilon}-1}{\beta_{j}^{\varepsilon} \lambda_{j}^{\varepsilon}}, \quad j=1,2,
$$

where we have set $\beta_{j}^{\varepsilon}=\int_{\mathbb{R}^{N}} \beta(x) \varphi_{j}^{\varepsilon}(x) \mathrm{d} x$. Notice that $\beta_{1}^{\varepsilon}>0$ (since $\varphi_{1}^{\varepsilon}>0$ and $\beta>0)$.

Then the large time behaviour of (2.5) is described in the next result. 
Theorem 2.2 (asymptotic behaviour) Let Assumption 2.1 be satisfied. Let $\varepsilon>0$ be given and fixed. Assume that the initial data satisfy $U_{0} \geq 0$ and $v_{0} \in L^{2}(\mathbb{R})$ with $v_{0} \geq 0$. Then the following holds true:

(i) If $\mathcal{R}_{0}^{\varepsilon} \leq 1$ or if $v_{0} \equiv 0$, the solution $(U, v)$ of $(2.5)$ converges in $\mathbb{R} \times L^{2}\left(\mathbb{R}^{N}\right)$ toward the disease free equilibrium, which reads as $(U(t), v(t,).) \rightarrow(\Lambda, 0)$ as $t \rightarrow \infty$.

(ii) If $\mathcal{R}_{0}^{\varepsilon}>1$ and $v_{0} \not \equiv 0$, the solution $(U, v)$ of (2.5) converges in $\mathbb{R} \times$ $L^{2}\left(\mathbb{R}^{N}\right)$ toward the endemic equilibrium, which reads as $(U(t), v(t,).) \rightarrow$ $\left(U_{1}^{\varepsilon}, V_{1}^{\varepsilon} \varphi_{1}^{\varepsilon}(\cdot)\right)$ as $t \rightarrow \infty$.

The above result describes the asymptotic behaviour of System (2.5) for any given and fixed $\varepsilon>0$. In order to go further in our analysis we shall make use of the asymptotic $\varepsilon \ll 1$ in order to describe the transient behaviour of the problem. To state our second main result we shall write our main assumptions in a rather abstract way based on the spectral elements of operator $L_{\varepsilon}$. These assumptions will then be discussed below in Remark 2.5.

As explained in the introduction we consider the case where the fitness function has two global maxima and the initial data is somehow well prepared. This precise assumption reads as follows.

Assumption 2.3 We assume that the fitness function satisfies

$$
\mathcal{S}=\left\{x \in \mathbb{R}^{N}: \Theta(x)=\max _{y \in \mathbb{R}^{N}} \Theta(y)\right\}=\left\{x_{1}, x_{2}\right\} \text { with } x_{1} \neq x_{2} .
$$

The initial data $\left(U_{0}, v_{0}\right) \in[0, \infty) \times\left(\mathcal{C}_{\mathrm{b}}^{0}\left(\mathbb{R}^{N}\right) \cap L_{+}^{2}\left(\mathbb{R}^{N}\right)\right)$ is such that there exists $\rho>0$ such that $v_{0} \equiv 0$ on $B\left(x_{1}, \rho\right)$, and $v_{0}\left(x_{2}\right)>0$. Here $B\left(x_{1}, \rho\right)$ denotes the open ball of radius $\rho$ centred at $x_{1}$ and $\mathcal{C}_{\mathrm{b}}^{0}\left(\mathbb{R}^{N}\right)$ the set of the continuous and bounded functions on $\mathbb{R}^{N}$.

Our next assumption is related to both the mutation kernel and the fitness function. As previously mentioned it is written in a rather abstract way using the three first eigen-elements associated to $L_{\varepsilon}$. It reads as follows.

Assumption 2.4 We assume that the following properties hold true.

(i) Spectral gap: the spectral gaps $G(\varepsilon)$ and $H(\varepsilon)$ defined by

$$
G(\varepsilon):=\lambda_{1}^{\varepsilon}-\lambda_{2}^{\varepsilon} \text { and } H(\varepsilon):=\lambda_{2}^{\varepsilon}-\lambda_{3}^{\varepsilon},
$$

satisfy for $\varepsilon \ll 1: H(\varepsilon)=h(\varepsilon) \varepsilon$ with $h(\varepsilon) \rightarrow h>0$ and $G(\varepsilon)|\ln \varepsilon| \ll \varepsilon$.

(ii) Concentration: For all $\nu>0$ one has for any $i=1,2$

$$
\int_{\mathbb{R}^{N} \backslash B\left(x_{i}, \nu\right)}\left|\varphi_{i}^{\varepsilon}\right| \mathrm{d} x=C(\nu, \varepsilon) \text { with } C(\nu, \varepsilon)=o\left(\varepsilon^{\infty}\right) \text { as } \varepsilon \rightarrow 0,
$$

and for each continuous and bounded function $f \in \mathcal{C}_{\mathrm{b}}^{0}\left(\mathbb{R}^{N}\right)$ one has

$$
\lim _{\varepsilon \rightarrow 0} \int_{\mathbb{R}^{N}} f(x) \frac{\varphi_{2}^{\varepsilon}(x)}{\left\|\varphi_{2}^{\varepsilon}\right\|_{L^{1}\left(\mathbb{R}^{N}\right)}} \mathrm{d} x=f\left(x_{2}\right) .
$$


Remark 2.5 In [9] the authors have proposed sufficient conditions, formulated in terms of decay rate for $J$ and local shape of $\Theta$ around $x_{1}$ and $x_{2}$, ensuring that the above assumption is satisfied. In particular, the main difference between the situations considered in Figures 1, 2 and 3 in the introduction, relies on the behaviour as $\varepsilon \rightarrow 0$ of the first spectral gap $G(\varepsilon)=\lambda_{1}^{\varepsilon}-\lambda_{2}^{\varepsilon}$. It follows from [9] (see Theorems 2.2 and 7.1 in the aforementioned work) that the eigenvalues $\lambda_{j}^{\varepsilon}$, for $j=1,2$, have an asymptotic expansion of the form

$$
\frac{\lambda_{j}^{\varepsilon}}{\|\Psi\|_{\infty}}=1+\lambda_{1, j} \varepsilon+\lambda_{2, j} \varepsilon^{2}+O\left(\varepsilon^{3}\right) \text { with } \lambda_{1, j}=-\frac{\sqrt{-\Psi^{\prime \prime}\left(x_{j}\right)}}{\sqrt{2\|\Psi\|_{\infty}}},
$$

while the coefficients $\lambda_{l, j}$ depend the successive derivatives of the function $\Psi$, namely $\Psi^{(m)}\left(x_{j}\right)$ with $m \geq 2$. So, the configuration in Figure 2, namely $\Psi^{\prime \prime}\left(x_{1}\right) \neq \Psi^{\prime \prime}\left(x_{2}\right)$, ensures that Assumption 2.4 is satisfied expect for the first spectral gap $G(\varepsilon)$ that is of order $\varepsilon$. On the other hand, the configuration of Figure 3, namely $\Psi^{\prime \prime}\left(x_{1}\right)=\Psi^{\prime \prime}\left(x_{2}\right)$, ensures that $\lambda_{1,1}=\lambda_{1,2}$ and $\lambda_{2,1}=\lambda_{2,2}$ (see [9] for the computation of this last coefficient), so that Assumption 2.4 is satisfied with $G(\varepsilon)=O\left(\varepsilon^{3}\right)$ as $\varepsilon \ll 1$. This difference in the first spectral gaps associated with the two configurations of Figures 2 and 3 roughly explain the change of order of magnitude observed in the duration of the transient regimes in these two numerical experiments.

In view of Theorem 2.2, the phenotype value $x_{1}$ is the 'stronger' EA since the infected population concentrates around $x_{1}$ as $t \rightarrow \infty$ and for $\varepsilon \ll 1$ small enough. Assumption 2.3 above means that the initial infected population is far from this 'stronger' phenotype value, $x_{1}$, while it displays a non negligible contribution on $x_{2}$. What we have in mind, as in the numerical experiments presented in the introduction, corresponds to an initial distribution of the infection with phenotypes concentrated around $x_{2}$, so that the initial infected population is mostly well adapted to this peak of the fitness. In that case, our next main result describes that the infected population exhibits a very long transient regime during which it stays concentrated around the phenotype value $x_{2}$ when $\varepsilon>0$ is small enough. This long duration turns out to be related to the first spectral gap $G(\varepsilon)$.

Now, before going to our second main result, note that it is easy to check, using Rayleigh quotient formulation, that

$$
\lambda_{1}^{\varepsilon} \rightarrow\|\Theta\|_{\infty}^{2} \text { as } \varepsilon \rightarrow 0 .
$$

Hence because of the spectral gap assumption $(i)$ described above, one also has

$$
\lambda_{2}^{\varepsilon} \rightarrow\|\Theta\|_{\infty}^{2} \text { as } \varepsilon \rightarrow 0 .
$$

In the sequel we set $\mathcal{R}_{0}^{0}$ the number defined (recalling (2.7)) by

$$
\mathcal{R}_{0}^{0}=\frac{\Lambda}{\mu}\|\Theta\|_{\infty}^{2}=\lim _{\varepsilon \rightarrow 0} \mathcal{R}_{0, j}^{\varepsilon}, j=1,2 .
$$


Let us also observe that the concentration assumptions (ii) imply that (see Remark 4.2 below)

$$
\frac{1}{\left\|\varphi_{1}^{\varepsilon}\right\|_{L^{1}}} \varphi_{1}^{\varepsilon} \rightarrow \delta_{x_{1}} \text { and } \frac{1}{\left\|\varphi_{2}^{\varepsilon}\right\|_{L^{1}}} \varphi_{2}^{\varepsilon} \rightarrow \delta_{x_{2}} \text { as } \varepsilon \rightarrow 0,
$$

where $\delta_{x_{i}}$ denotes the Dirac delta function at $x_{i}$. From the above concentration property one may observe that

$$
\beta_{2}^{\varepsilon}=\int_{\mathbb{R}^{N}} \beta \varphi_{2}^{\varepsilon} \mathrm{d} x \sim\left\|\varphi_{2}^{\varepsilon}\right\|_{L^{1}} \beta\left(x_{2}\right)>0 \text { as } \varepsilon \rightarrow 0 .
$$

We are now able to state our second main result.

Theorem 2.6 (transient regime) Let Assumptions 2.1, 2.3 and 2.4 be satisfied. Assume that $\mathcal{R}_{0}^{0}>1$ and recall (2.8). Then, for any $q>0$, there exist some positive constants $\ell$ and $c>0$ such that for each $\varepsilon>0$ small enough one has

$$
\left|U^{\varepsilon}(t)-U_{2}^{\varepsilon}\right|+\left\|v^{\varepsilon}(t, .)-V_{2}^{\varepsilon} \varphi_{2}^{\varepsilon}(.)\right\|_{L^{2}\left(\mathbb{R}^{N}\right)} \leq c \varepsilon^{q}, \forall t \in\left[\frac{\ell|\ln \varepsilon|}{\varepsilon}, \frac{1}{G(\varepsilon)}-\frac{|\ln \varepsilon|}{\varepsilon}\right] .
$$

Here one may notice that the point $\left(U_{2}^{\varepsilon}, V_{2}^{\varepsilon} \varphi_{2}^{\varepsilon}\right)$ is an equilibrium point of the system but it is not admissible. Indeed, although $U_{2}^{\varepsilon}>0$ and $V_{2}^{\varepsilon}>0$ for $\varepsilon \ll 1$, since $\lambda_{2}^{\varepsilon}$ is the second eigenvalue, Perron-Frobenius theorem implies that the eigenvector $\varphi_{2}^{\varepsilon}$ is not positive. In that sense it can be viewed as a quasiequilibrium point of System (2.5) when equipped with non-negative initial data. The above result describes the transient behaviour of the system around this quasi-equilibrium point. Moreover, because of Assumption $2.4(i)$ the duration of this transient regime is large in the sense that $\frac{1}{G(\varepsilon)}-\frac{(\ell+1)|\ln \varepsilon|}{\varepsilon} \rightarrow \infty$ as $\varepsilon \rightarrow 0$.

The proof of the above two results is based on a splitting argument of Problem (2.5) using the spectral decomposition of the operator $L_{\varepsilon}$. Indeed, for each given and fixed $\varepsilon>0$, we project the system on the Hilbert basis $\left\{\varphi_{i}^{\varepsilon}\right\}_{i \geq 1}$. More precisely we set

$$
v_{n}(t)=v_{n}^{\varepsilon}(t)=\int_{\mathbb{R}^{N}} v(t, x) \varphi_{n}^{\varepsilon}(x) \mathrm{d} x, \beta_{n}=\beta_{n}^{\varepsilon}=\int_{\mathbb{R}^{N}} \beta(x) \varphi_{n}^{\varepsilon}(x) \mathrm{d} x .
$$

Then System (2.5) re-writes as the following infinite system of ODE

$$
\left\{\begin{array}{l}
U^{\prime}(t)=\Lambda-U(t)-U(t) \sum_{n=1}^{\infty} \beta_{n} \lambda_{n}^{\varepsilon} v_{n}(t) \\
v_{n}^{\prime}(t)=v_{n}(t)\left(U(t) \lambda_{n}^{\varepsilon}-\mu\right), n \geq 1
\end{array}\right.
$$

with the following initial data

$$
U(0)=U_{0}, \quad v_{n}(0)=v_{0, n}^{\varepsilon}=\int_{\mathbb{R}^{N}} v_{0}(x) \varphi_{n}^{\varepsilon}(x) \mathrm{d} x, n \geq 1 .
$$


The proofs of Theorems 2.2 and 2.6 are strongly based on this re-formulation. Roughly speaking we will show that higher order modes (for $n \geq 3$ ) become very small for $t \geq \frac{1}{\varepsilon}$ so that the behaviour of the system reduces to the two first modes in (2.9).

Finally let us mention that the transition from the almost stationary transient regime, described in Theorem 2.6, and the large time behaviour, described in Theorem 2.2, will not be studied in this paper and let for future work. Here since $v_{n}$ with $n \geq 3$ is very small for $t \gg 1 / \varepsilon$ and $\varepsilon \ll 1$, we suspect that this transition regime is related to heteroclinic orbits for the three dimensional system $(2.9)$ for $\left(U, v_{1}, v_{2}\right)$ and $v_{n}=0$ for $n \geq 3$.

\section{Proof of Theorem 2.2}

Since the results stated in Theorem 2.2 do not directly depend on $\varepsilon$, throughout this section we fix $\varepsilon>0$ and for the sake of simplicity we omit to explicitly write down the superscripts $\varepsilon$.

\subsection{Preliminary estimates}

Here we derive preliminary estimates that will be needed for the proof of Theorem 2.2 .

The first lemma is an identity between the modes arising in the $v$-component of the solution of (2.5). It will be used at various places in this article, along the proof of Theorem 2.2 and also for the one of Theorem 2.6.

Lemma 3.1 For any $m, n \in \mathbb{N}$ the following identity holds true

$$
\forall t \geq 0, \quad v_{m}(t)=v_{0, m}\left(\frac{v_{n}(t)}{v_{0, n}}\right)^{\lambda_{m} / \lambda_{n}} e^{-\frac{\lambda_{n}-\lambda_{m}}{\lambda_{n}} \mu t},
$$

whenever $v_{0, n} \neq 0$.

Proof of Lemma 3.1. Integrating the equation for $v_{m}$ yields

$$
v_{m}(t)=v_{0, m} e^{\int_{0}^{t} \lambda_{m} U(s) \mathrm{d} s-\mu t}=v_{0, m}\left\{\exp \left(\int_{0}^{t} \lambda_{n} U(s) \mathrm{d} s-\mu t\right)\right\}^{\frac{\lambda_{m}}{\lambda_{n}}} e^{\frac{\lambda_{m}-\lambda_{n}}{\lambda_{n}} \mu t},
$$

and the result follows.

We now derive some upper bounds for the solutions of (2.9). These estimates will be used to prove Theorem 2.2. Some other refined estimates will be needed for the proof of Theorem 2.6 since the ones described below may depend on $\varepsilon$.

Proposition 3.2 Let $\left(U(t), v_{n}(t) ; n \geq 1\right)_{t>0}$ be the solution of (2.9) with initial data $\left(U_{0}, v_{0, n} ; n \geq 1\right)$. Let us recall that $\beta_{1}>0$. Then we have the following estimates 
1. Let $P(t)=U(t)+\int_{\mathbb{R}^{N}} \beta(x) v(t, x) \mathrm{d} x$, then

$$
\forall t \geq 0, U(t) \leq \max \left(\Lambda, U_{0}\right) \text { and } P(t) \leq \Lambda+(P(0)-\Lambda) e^{-t} .
$$

2. There exists a positive constant $C$ such that

$$
U(t)+\frac{\beta_{1} v_{1}(t)}{2} \leq \max \left\{U_{0}+\frac{\beta_{1} v_{0,1}}{2}, C\right\}, \forall t \geq 0 .
$$

3. Assume that $v_{1,0}>0$. Set

$$
w_{2}(t, x)=\sum_{n=2}^{+\infty} v_{n}(t) \varphi_{n}(x) \text { and } R_{2}(t)=\sum_{n=2}^{+\infty} \lambda_{n} \beta_{n} v_{n}(t) .
$$

Then the following estimate holds true

$$
\max \left\{\left\|w_{2}(t, .)\right\|_{L^{2}(\mathbb{R})},\left|R_{2}(t)\right|\right\} \leq c e^{-\frac{\lambda_{1}-\lambda_{2}}{\lambda_{1}} \mu t}, \quad \forall t \geq 0 .
$$

Proof. First note that the solution $(U, v)$ of $(2.5)$ is non-negative, then $L_{\varepsilon}[v]$ is non-negative and the first inequality follows. Next, combining both equations of (2.5) and noticing that $\mu \geq 1$ readily implies that $P^{\prime}(t) \leq \Lambda-P(t), \forall t \geq 0$, which proves 1 .

Next if $v_{0,1}=0$ the inequality is trivial. If $v_{0,1}>0$ then $v_{1}(t)>0$ for all $t \geq 0$. Next the $U$-equation of system (2.9) rewrites

$$
U^{\prime}(t)=\Lambda-U(t)-U(t)\left(\lambda_{1} \beta_{1} v_{1}(t)+\sum_{n=2}^{\infty} \lambda_{n} \beta_{n} v_{n}(t)\right)
$$

hence setting $B(t)=U(t)+\frac{1}{2} \beta_{1} v_{1}(t)$, then as $R_{2}(t)=\sum_{n=2}^{+\infty} \lambda_{n} \beta_{n} v_{n}(t)$, using the $v_{1}$ equation of (2.9) we find that for all $t \geq 0$

$$
B^{\prime}(t)=\Lambda-U(t)-\frac{1}{2} \lambda_{1} \beta_{1} v_{1}(t) U(t)-R_{2}(t) U(t)-\frac{1}{2} \beta_{1} \mu v_{1}(t) .
$$

We now estimate $w_{2}(t, x)$ and $R_{2}(t)$. Let $n \geq 2$ be fixed. By integrating the $v_{n}$-equation for system $(2.9)$ we find

$$
v_{n}(t)=v_{0, n} e^{\int_{0}^{t} \lambda_{n} U(s) \mathrm{d} s-\mu t},
$$

then since $\lambda_{n} \leq \lambda_{2}$ we get

$$
\left|v_{n}(t)\right| \leq\left|v_{0, n}\right| e^{\int_{0}^{t} \lambda_{2} U(s) \mathrm{d} s-\mu t},
$$

and, as for the proof of Lemma 3.1, we readily establish

$$
\left|v_{n}(t)\right| \leq\left|v_{0, n}\right|\left(\frac{v_{1}(t)}{v_{0,1}}\right)^{\frac{\lambda_{2}}{\lambda_{1}}} e^{-\frac{\lambda_{1}-\lambda_{2}}{\lambda_{1}} \mu t} .
$$


As a consequence, the following estimates hold

$$
\left\|w_{2}(t, .)\right\|_{L^{2}(\mathbb{R})} \leq\left\|v_{0}\right\|_{L^{2}(\mathbb{R})}\left(\frac{v_{1}(t)}{v_{0,1}}\right)^{\frac{\lambda_{2}}{\lambda_{1}}} e^{-\frac{\lambda_{1}-\lambda_{2}}{\lambda_{1}} \mu t},
$$

and

$$
\left|R_{2}(t)\right| \leq \lambda_{1}\|\beta\|_{L^{2}(\mathbb{R})}\left\|v_{0}\right\|_{L^{2}(\mathbb{R})}\left(\frac{v_{1}(t)}{v_{0,1}}\right)^{\frac{\lambda_{2}}{\lambda_{1}}} e^{-\frac{\lambda_{1}-\lambda_{2}}{\lambda_{1}} \mu t} .
$$

Next let $k>0$ be some constant to be chosen later. Since $\beta_{1}>0$, Young's inequality ensures that

$$
\left(\frac{v_{1}(t)}{v_{0,1}}\right)^{\frac{\lambda_{2}}{\lambda_{1}}} \leq \frac{\lambda_{2}}{\lambda_{1}} \frac{\beta_{1} v_{1}(t)}{k}+\frac{\lambda_{1}-\lambda_{2}}{\lambda_{1}}\left(\frac{k}{\beta_{1} v_{0,1}}\right)^{\frac{\lambda_{2}}{\lambda_{1}-\lambda_{2}}},
$$

Coming back to (3.12), thanks to the non negativity of $\lambda_{1} \beta_{1} v_{1}$, we first write

$$
B^{\prime}(t) \leq \Lambda-U(t)-R_{2}(t) U(t)-\frac{1}{2} \beta_{1} \mu v_{1}(t),
$$

then writing $-R_{2} \leq\left|R_{2}\right|$ and using estimates (3.10), (3.14), and (3.15), we obtain the following estimate

$$
\begin{aligned}
B^{\prime}(t) \leq & \Lambda-U(t)+\left\{\frac{\lambda_{2}}{k} \max \left(\Lambda, U_{0}\right)\|\beta\|_{L^{2}(\mathbb{R})}\left\|v_{0}\right\|_{L^{2}(\mathbb{R})}-\frac{\mu}{2}\right\} \beta_{1} v_{1}(t) \\
& +\left(\lambda_{1}-\lambda_{2}\right)\left(\frac{k}{\beta_{1} v_{0,1}}\right)^{\frac{\lambda_{2}}{\lambda_{1}-\lambda_{2}}}\|\beta\|_{L^{2}(\mathbb{R})}\left\|v_{0}\right\|_{L^{2}(\mathbb{R})} \max \left(\Lambda, U_{0}\right),
\end{aligned}
$$

so that choosing $k=\frac{4 \lambda_{2} \max \left(\Lambda, U_{0}\right)\|\beta\|_{L^{2}(\mathbb{R})}\left\|v_{0}\right\|_{L^{2}(\mathbb{R})}}{\mu}$, and setting

$$
d=\min \left\{1, \frac{\mu}{4}\right\}
$$

and

$$
f=\Lambda+\left(\lambda_{1}-\lambda_{2}\right)\left(\frac{k}{\beta_{1} v_{0,1}}\right)^{\frac{\lambda_{2}}{\lambda_{1}-\lambda_{2}}}\|\beta\|_{L^{2}(\mathbb{R})}\left\|v_{0}\right\|_{L^{2}(\mathbb{R})} \max \left(\Lambda, U_{0}\right),
$$

we find $B^{\prime}(t) \leq f-d B(t)$, hence

$$
B(t)=U(t)+\frac{\beta_{1} v_{1}(t)}{2} \leq \max \left\{U_{0}+\frac{\beta_{1} v_{0,1}}{2}, \frac{f}{d}\right\},
$$

for all $t \geq 0$. This ends the proof of 2 .

Finally combining (3.13), (3.14) and the previous result yields (3.11) and completes the proof of the lemma. 


\subsection{Proof of Theorem 2.2}

Due to (3.11), let us observe that $\left\|v(t, .)-v_{1}(t) \varphi_{1}(\cdot)\right\|_{L^{2}(\mathbb{R})}$ tends to 0 as $t \rightarrow \infty$, therefore the asymptotic behaviour of $(U, v)$ is given by the one of the solution $\left(U, v_{1}\right)$ of $(2.9)$ which satisfies the following system of equations

$$
\left\{\begin{array}{l}
U^{\prime}(t)=\Lambda-U(t)-\lambda_{1} \beta_{1} v_{1}(t) U(t)-R_{2}(t) U(t), \\
v_{1}^{\prime}(t)=v_{1}(t)\left(\lambda_{1} U(t)-\mu\right) .
\end{array}\right.
$$

which is asymptotically autonomous since, thanks to (3.14), we have $R_{2}(t) \rightarrow 0$ as $t \rightarrow \infty$.

Next let us first recall a result for the limit system associated to (3.16).

Lemma 3.3 Consider the system

$$
\left\{\begin{array}{l}
V^{\prime}(t)=\Lambda-V(t)-\lambda_{1} \beta_{1} w(t) V(t) \\
w^{\prime}(t)=w(t)\left(\lambda_{1} V(t)-\mu\right)
\end{array}\right.
$$

Then the following properties hold true:

(i) If $\mathcal{R}_{0}=\frac{\lambda_{1} \Lambda}{\mu} \leq 1$ then the above system has a unique bounded and non negative complete orbit $(V(t), w(t)) \equiv(\Lambda, 0)$ for all $t \in \mathbb{R}$.

(ii) Assume $\mathcal{R}_{0}>1$. Let $(V(t), w(t))_{t \in \mathbb{R}}$ be a bounded non negative complete orbit. Then one has

$$
\inf _{t \in \mathbb{R}} w(t)>0 \Longrightarrow(V(t), w(t)) \equiv\left(\frac{\mu}{\lambda_{1}}, \frac{\mathcal{R}_{0}-1}{\lambda_{1} \beta_{1}}\right), \forall t \in \mathbb{R} .
$$

(iii) Assume $\mathcal{R}_{0}>1$. Let $(V(t), w(t))_{t \geq 0}$ be a bounded and non negative orbit. Then one has

$$
\exists t_{0} \geq 0, w\left(t_{0}\right)>0 \Longrightarrow \lim _{t \rightarrow \infty}(V(t), w(t))=\left(\frac{\mu}{\lambda_{1}}, \frac{\mathcal{R}_{0}-1}{\lambda_{1} \beta_{1}}\right) .
$$

The proof of this result is based on standard Lyapunov arguments. We refer for instance to [10] for the proof of this lemma (with additional diffusion terms).

We now split our arguments into two parts. We first consider the case $\mathcal{R}_{0} \leq 1$ and then we will investigate the case $\mathcal{R}_{0}>1$.

The case $\mathcal{R}_{0} \leq 1$ : let us show that $\left(U(t), v_{1}(t)\right) \rightarrow(\Lambda, 0)$ as $t \rightarrow \infty$. To that aim, let $\left\{t_{n}\right\}_{n \geq 0}$ be a given sequence such that $t_{n} \rightarrow \infty$. Consider the sequence of functions $\left(\bar{U}_{n}(t), w_{n}(t)\right)=\left(U, v_{1}\right)\left(t+t_{n}\right)$.

Since $\left(U, v_{1}\right)$ is bounded in the $C^{1}$-norm due to proposition 3.2 and System (3.16), one may assume possibly along a subsequence that $\left(U_{n}, w_{n}\right)(t) \rightarrow$ $(V, w)(t)$ locally uniformly for $t \in \mathbb{R}$ and $(V, w)$ is a bounded and non-negative complete orbit of the autonomous problem (3.17).

However since $\mathcal{R}_{0} \leq 1$, Lemma 3.3 applies and ensures $(V(t), w(t)) \equiv(\Lambda, 0)$. This completes the first part of the result. 
The case $\mathcal{R}_{0}>1$ : let us first notice that if $v_{0} \equiv 0$ (or equivalently $v_{0,1}=0$ ) then $v_{1}(t)=0$ for all $t \geq 0$ and we obviously obtain $\left(U(t), v_{1}(t)\right) \rightarrow(\Lambda, 0)$ as $t \rightarrow \infty$.

We now consider the case $v_{0} \not \equiv 0$, that is $v_{0,1}>0$. To that aim let us define

$$
\mathcal{M}_{0}=\left\{\left(U_{0}, v_{0,1}\right) \in\left(\mathbb{R}^{+}\right)^{2}, v_{0,1}>0\right\} .
$$

Then the following lemma holds true

Lemma 3.4 A uniform weak persistence property holds for System (3.16) in the following sense

$$
\forall\left(U_{0}, v_{0,1}\right) \in \mathcal{M}_{0}, \limsup _{t \rightarrow+\infty} v_{1}(t) \geq V_{1}^{*} \text { with } V_{1}^{*}=\frac{\mathcal{R}_{0}-1}{\lambda_{1} \beta_{1}} .
$$

Proof. Indeed if by contradiction we assume that there exists $\eta \in\left(0, V_{1}^{*}\right)$ and $\left(U_{0}, v_{0,1}\right)$ with $v_{0,1}>0$ such that $\lim \sup _{t \rightarrow+\infty} v_{1}(t)<\eta$, then there exists $t_{1}>0$ such that $\forall t \geq t_{1}, v_{1}(t) \leq \eta$. Moreover, for any $s$ and $t$ such that $t \geq s \geq t_{1}$ we have

$$
v_{1}(t)=v_{1}(s) e^{\int_{s}^{t}\left(\lambda_{1} U(r)-\mu\right) \mathrm{d} r} .
$$

Let us denote $\underline{U}=\liminf _{t \rightarrow+\infty} U(t)$. Let $\alpha>0$ to be chosen later, there exists $t_{2}(\alpha) \geq t_{1}$ such that

$$
\forall t, s \in \mathbb{R}, t \geq s \geq t_{2} \Longrightarrow v_{1}(t) \geq v_{1}(s) e^{\int_{s}^{t}\left(\lambda_{1}(\underline{U}-\alpha)-\mu\right) \mathrm{d} s},
$$

then as $v_{1}$ is bounded and positive on $\mathbb{R}^{+}$we have the following necessary condition on $\underline{U}$

$$
\underline{U} \leq \frac{\mu}{\lambda_{1}}+\alpha .
$$

Next let $\left(t_{n}\right)_{n \geq 0}$ be a sequence that tends to $\infty$ as $n \rightarrow \infty$ and such that $\lim _{n \rightarrow+\infty} U^{\prime}\left(t_{n}\right)=0$ and $\lim _{n \rightarrow+\infty} U\left(t_{n}\right)=\underline{U}$, as $v_{1}\left(t_{n}\right) \leq \eta$ for $n$ large enough we have

$$
U^{\prime}\left(t_{n}\right) \geq \Lambda-U\left(t_{n}\right)-\lambda_{1} \beta_{1} \eta U\left(t_{n}\right)-R_{2}\left(t_{n}\right) U\left(t_{n}\right),
$$

passing to the limit this gives

$$
\underline{U} \geq \frac{\Lambda}{1+\lambda_{1} \beta_{1} \eta} .
$$

Now as $\eta<V_{1}^{*}=\frac{\mathcal{R}_{0}-1}{\lambda_{1} \beta_{1}}$, then $\mathcal{R}_{0} \frac{1}{1+\lambda_{1} \beta_{1} \eta}>1$ and we can choose $\alpha>0$ such that $\alpha \frac{\lambda_{1}}{\mu}<\mathcal{R}_{0} \frac{1}{1+\lambda_{1} \beta_{1} \eta}-1$. This choice implies that

$$
\frac{\Lambda}{1+\lambda_{1} \beta_{1} \eta}>\frac{\mu}{\lambda_{1}}+\alpha
$$

which is absurd since (3.18) and (3.19), and Lemma 3.4 is proved.

Next we have the following result 
Lemma 3.5 A strong persistence property holds for System (3.16) in the following sense

$$
\forall\left(U_{0}, v_{0,1}\right) \in \mathcal{M}_{0}, \liminf _{t \rightarrow+\infty} v_{1}(t)>0 .
$$

Proof. Assume by contradiction that for some initial condition $\left(U_{0}, v_{0,1}\right) \in \mathcal{M}_{0}$, $\liminf _{t \rightarrow+\infty} v_{1}(t)=0$. Due to Lemma 3.4 there exists a sequence $\left(t_{n}\right)$ that tends to infinity such that $\forall n, v_{1}\left(t_{n}\right) \geq \eta$, for some $\eta \in\left(0, V_{1}^{*}\right)$. Then there exists a sequence $\left(T_{n}\right)$ with $T_{n}>t_{n}$ for all $n$ and $\lim _{n \rightarrow+\infty} v_{1}\left(T_{n}\right)=0$. As $v_{1}$ is continuous, we define

$$
h_{n}=\inf \left\{h \in\left(0, T_{n}-t_{n}\right), v_{1}\left(T_{n}-h\right)=\eta / 2\right\} .
$$

Let us set $X(t)=\left(U(t), v_{1}(t)\right)$. We define for all $t \geq-T_{n}$ the sequence of solutions $Y_{n}(t)=X\left(t+T_{n}\right)$. As in the case $\mathcal{R}_{0} \leq 1$, possibly up to a subsequence, as $n$ tends to infinity, $\left\{Y_{n}(t)\right\}_{t \geq-T_{n}}$ converges - locally uniformly - toward a complete orbit $\{Y(t)=(V(t), w(t))\}_{t \in \mathbb{R}}$ of the autonomous system (3.17). Moreover as $\lim _{n \rightarrow+\infty} v_{1}\left(T_{n}\right)=0$ then $w(0)=0$, hence $\forall t \in \mathbb{R}, w(t)=0$. Assume now that $\left(h_{n}\right)$ is bounded from above then up to a sub-sequence $\left(h_{n}\right)$ converges toward some $\bar{h} \geq 0$ and $w(-\bar{h})=\eta / 2>0$, a contradiction. Hence up to a subsequence $\lim _{n \rightarrow+\infty} h_{n}=+\infty$.

Similarly we define $Y_{n}(t)=X\left(t+T_{n}-h_{n}\right), \forall t \geq-T_{n}+h_{n} \geq-t_{n}$ and consider its limit as $n$ tends to infinity, that is the complete orbit $\{Y(t)=$ $(V(t), w(t))\}_{t \in \mathbb{R}}$ of system (3.17). Then $w(0)=\eta / 2$ and by definition of $\left(h_{n}\right)$ and as $\lim _{n \rightarrow+\infty} h_{n}=+\infty$ we also have $\forall t \geq 0, w(t) \leq \eta / 2$, which is impossible. Indeed due to Lemma 3.3 (iii) a complete orbit of system (3.17) with $w(0)>0$ converges as $t \rightarrow+\infty$ toward the stationary orbit $\left(U_{1}^{*}, V_{1}^{*}\right)$ and $V_{1}^{*}>\eta / 2$. By contradiction the lemma is proved.

To conclude the proof of Theorem 2.2, consider a solution $X(t)=\left(U(t), v_{1}(t)\right)$ of system (3.16) for $t \in \mathbb{R}^{+}$associated to an initial condition in $\mathcal{M}_{0}$. As $X(t)$ is bounded on $\mathbb{R}^{+}$, there is a sequence $\left(t_{n}\right)_{n \geq 0}$ that tends to infinity and $\left(U^{*}, V^{*}\right) \in\left(\mathbb{R}^{+}\right)^{2}$ such that $\lim _{n \rightarrow+\infty} X\left(t_{n}\right)=\left(U^{*}, V^{*}\right)$, and thanks to Lemma $3.5, V^{*}>0$. Then if we are able to prove that $\left(U^{*}, V^{*}\right)=\left(U_{1}^{*}, V_{1}^{*}\right)$, then the proof of theorem 2.2 is complete.

To do so let us set $Y_{n}(t)=X\left(t+t_{n}\right), \forall t \geq-t_{n}$. As $n$ tends to infinity $\left\{Y_{n}(t)\right\}_{t \in \mathbb{R}}$ locally uniformly converges toward a complete orbit $\{Y(t)=$ $(V(t), w(t))\}_{t \in \mathbb{R}}$ of system (3.17) with $Y(0)=\left(U^{*}, V^{*}\right)$. Thanks to Lemma 3.5 there exists $\eta^{\prime}>0$ such that $\liminf _{n \rightarrow+\infty} v_{1}\left(t+t_{n}\right) \geq \eta^{\prime}$, hence $\forall t \in \mathbb{R}, w(t) \geq$ $\eta^{\prime}$. And Lemma $3.3($ ii $)$ applies and implies $Y(t) \equiv\left(U_{1}^{*}, V_{1}^{*}\right)$ and we necessarily have $\left(U^{*}, V^{*}\right)=\left(U_{1}^{*}, V_{1}^{*}\right)$. The proof is complete.

\section{Proof of Theorem 2.6}

Here again we split this section into two parts. We first derive some estimates by including the dependence upon the parameter $\varepsilon$ that we will be assumed to be small enough. The second part of this section focuses on the proof of Theorem 2.6 . 


\subsection{Estimates}

In this section we derive estimates with an explicit dependence on $\varepsilon$ for the proof of Theorem 2.6. Thus in this section and the next one we assume that Assumptions 2.1, 2.3 and 2.4 are satisfied.

We start with an estimate in $L^{1}\left(\mathbb{R}^{N}\right)$ of the eigenvectors $\varphi_{1}^{\varepsilon}$ and $\varphi_{2}^{\varepsilon}$ of the operator $L_{\varepsilon}$ associated to the first eigenvalues.

Lemma 4.1 The eigenvectors $\varphi_{1}^{\varepsilon}$ and $\varphi_{2}^{\varepsilon}$ satisfy the following estimates:

There exists a positive constant $C>0$ such that for all $\varepsilon \ll 1$ small enough one has for $i=1,2$

$$
C \varepsilon^{\frac{N}{2}} \leq\left\|\varphi_{i}^{\varepsilon}\right\|_{L^{1}\left(\mathbb{R}^{N}\right)} \text { and }\left\|\varphi_{i}^{\varepsilon}\right\|_{L^{1}\left(\mathbb{R}^{N}\right)}=o(1) \text { as } \varepsilon \rightarrow 0 .
$$

Proof. We start with the first estimate in (4.20). In the following $c$ denotes any positive constant that does not depend on the parameter $\varepsilon$. To that aim recall that for $i=1,2$, one has $\lambda_{i}^{\varepsilon} \varphi_{i}^{\varepsilon}=L_{\varepsilon}\left(\varphi_{i}^{\varepsilon}\right)$, while the operator $L_{\varepsilon}$ is given in (2.6). Since $\lambda_{i}^{\varepsilon}, \Theta, J_{\varepsilon}$ are non negative functions and since $\Theta$ is bounded on $\mathbb{R}$ (Assumption 2.3), we have

$$
\lambda_{i}^{\varepsilon}\left|\varphi_{i}^{\varepsilon}(x)\right| \leq \max _{y \in \mathbb{R}^{N}} \Theta^{2}(y) J_{\varepsilon} *\left|\varphi_{i}^{\varepsilon}\right|(y) .
$$

Next using Young's inequality to estimate the convolution product we obtain that

$$
\lambda_{i}^{\varepsilon}\left\|\varphi_{i}^{\varepsilon}\right\|_{L^{2}\left(\mathbb{R}^{N}\right)} \leq c\left\|J_{\varepsilon}\right\|_{L^{2}\left(\mathbb{R}^{N}\right)}\left\|\varphi_{i}^{\varepsilon}\right\|_{L^{1}\left(\mathbb{R}^{N}\right)},
$$

with $\lambda_{i}^{\varepsilon} \geq c>0$ for $\varepsilon \ll 1$. Next recalling the normalisation $\left\|\varphi_{i}^{\varepsilon}\right\|_{L^{2}\left(\mathbb{R}^{N}\right)}=1$ and thanks to Assumption 2.1, namely $J \in L^{1}\left(\mathbb{R}^{N}\right) \cap L^{\infty}\left(\mathbb{R}^{N}\right)$, we get

$$
\left\|J_{\varepsilon}\right\|_{L^{2}\left(\mathbb{R}^{N}\right)}=\left(\int_{\mathbb{R}^{N}} J_{\varepsilon}(x) \frac{1}{\varepsilon^{N}} J\left(\frac{x}{\varepsilon}\right) \mathrm{d} x\right)^{1 / 2} \leq c \varepsilon^{-\frac{N}{2}},
$$

and the estimate follows.

For the second estimate in (4.20), let us observe that using the concentration property stated in Assumption 2.4 (ii) we get for each $\nu>0$ and $\varepsilon$ small enough:

$$
\left\|\varphi_{i}^{\varepsilon}\right\|_{L^{1}\left(\mathbb{R}^{N}\right)}=\int_{B\left(x_{i}, \nu\right)}\left|\varphi_{i}^{\varepsilon}\right| \mathrm{d} x+\int_{\mathbb{R}^{N} \backslash B\left(x_{i}, \nu\right)}\left|\varphi_{i}^{\varepsilon}\right| \mathrm{d} x \leq \int_{B\left(x_{i}, \nu\right)}\left|\varphi_{i}^{\varepsilon}\right| \mathrm{d} x+C(\nu, \varepsilon) .
$$

Next Cauchy-Schwarz inequality yields

$$
\int_{B\left(x_{i}, \nu\right)}\left|\varphi_{i}^{\varepsilon}\right| \mathrm{d} x \leq\left(\int_{B\left(x_{i}, \nu\right)} 1 \mathrm{~d} x\right)^{1 / 2}\left\|\varphi_{i}^{\varepsilon}\right\|_{L^{2}\left(\mathbb{R}^{N}\right)} \leq c \nu^{N / 2} .
$$

As a consequence for each $\nu>0$ one gets

$$
\limsup _{\varepsilon \rightarrow 0}\left\|\varphi_{i}^{\varepsilon}\right\|_{L^{1}\left(\mathbb{R}^{N}\right)} \leq c \nu^{N / 2},
$$

and the result follows letting $\nu \rightarrow 0$. 
Remark 4.2 Note that Assumption 2.4 (ii) and the above theorem implies that for any $\nu>0$ one has

$$
\lim _{\varepsilon \rightarrow 0} \int_{\mathbb{R}^{N} \backslash B\left(x_{1}, \nu\right)} \frac{\varphi_{1}^{\varepsilon}}{\left\|\varphi_{1}^{\varepsilon}\right\|_{L^{1}\left(\mathbb{R}^{N}\right)}} \mathrm{d} x=0 .
$$

Since $\varphi_{1}^{\varepsilon}>0$ this implies that $\frac{\varphi_{1}^{\varepsilon}}{\left\|\varphi_{1}^{\varepsilon}\right\|_{L^{1}\left(\mathbb{R}^{N}\right)}} \rightarrow \delta_{x_{1}}$ as $\varepsilon \rightarrow 0$ for the narrow topology.

Next we give estimates for the initial conditions $v_{0,1}, v_{0,2}$ and for the coefficients $\beta_{1}, \beta_{2}$.

Lemma 4.3 There exists some constant $c>0$ such that the initial condition $v_{0,1}=v_{0,1}^{\varepsilon}$ satisfies the following estimates for all $\varepsilon$ small enough

$$
0<v_{0,1} \leq c C(\rho, \varepsilon),
$$

where $\rho>0$ is defined in Assumption 2.3 and $C(\rho, \varepsilon)$ is defined in Assumption 2.4 (ii). Moreover there exists $c>0$ such that for all $\varepsilon \ll 1$ one has

$$
c \varepsilon^{\frac{N}{2}} \leq v_{0,2} \leq o(1)
$$

Lastly, coefficients $\beta_{1}$ and $\beta_{2}$ satisfy

$$
c \varepsilon^{\frac{N}{2}}<\beta_{1} \leq o(1), c \varepsilon^{\frac{N}{2}}<\beta_{2} \leq o(1),
$$

with $c$ a positive constant independent of $\varepsilon$.

Proof. First note that since $v_{0} \geq 0$ and $v_{0} \not \equiv 0$ then $v_{0,1}>0$ since $\varphi_{1}^{\varepsilon}>0$. Next because of Assumption 2.3 on the initial condition $v_{0}$ one also has

$$
v_{0,1}=\int_{\mathbb{R}^{N} \backslash B\left(x_{1}, \rho\right)} v_{0}(x) \varphi_{1}^{\varepsilon}(x) \mathrm{d} x \leq\left\|v_{0}\right\|_{\infty} C(\rho, \varepsilon) .
$$

Hence (4.21) follows.

Next since $v_{0}$ is continuous and $v_{0}\left(x_{2}\right)>0$, one obtains due to Assumption $2.4(i i)$

$$
v_{0,2}^{\varepsilon}=\left\|\varphi_{2}^{\varepsilon}\right\|_{L^{1}\left(\mathbb{R}^{N}\right)} \int_{\mathbb{R}^{N}} v_{0}(x) \frac{\varphi_{2}^{\varepsilon}(x)}{\left\|\varphi_{2}^{\varepsilon}\right\|_{L^{1}\left(\mathbb{R}^{N}\right)}} \mathrm{d} x \sim\left\|\varphi_{2}^{\varepsilon}\right\|_{L^{1}\left(\mathbb{R}^{N}\right)} v_{0}\left(x_{2}\right) \text { as } \varepsilon \rightarrow 0 .
$$

Hence (4.22) follows from (4.20).

Finally since $\beta(x)>0$ for all $x \in \mathbb{R}^{N}$, so that $\beta\left(x_{1}\right)>0$ and $\beta\left(x_{2}\right)>0$, (4.23) follows from a similar argument as above using (4.20), Assumption 2.4 (ii) and Remark 4.2 .

We now give an upper bound of the solution that doesn't depend on $\varepsilon$, after some transition phase. 
Lemma 4.4 (Upper bound) For $k \in(0,+\infty)$, we set $t_{k}^{\varepsilon}=\frac{k}{\varepsilon} \ln \left(\frac{1}{\varepsilon}\right)$. Let us consider the function $Q=Q^{\varepsilon}=U+\beta_{1}^{\varepsilon} v_{1}+\frac{1}{2} \beta_{2}^{\varepsilon} v_{2}$. There exist positive constants $c$ and $k_{1}$ such that for all $\varepsilon>0$ small enough the following upper bound holds true

$$
0<Q(t) \leq c, \forall t \geq t_{k_{1}}^{\varepsilon} .
$$

Proof. First recall that $U>0, \beta_{1}>0$ and $v_{1}>0$ while $\beta_{2}>0$ and $v_{0,2}>0$ for $\varepsilon \ll 1$, so that $v_{2}(t)>0$ for any $t \geq 0$ as soon as $\varepsilon$ is small enough. Hence $Q(t)>0$ for $\varepsilon$ small enough.

Next let $R_{3}(t)=\sum_{n=3}^{+\infty} \lambda_{n} \beta_{n} v_{n}(t)$. Thanks to similar computations as the ones performed to prove (3.14) we readily establish

$$
\left|R_{3}(t)\right| \leq\left|\lambda_{3}\right|\|\beta\|_{L^{2}(\mathbb{R})}\left\|v_{0}\right\|_{L^{2}(\mathbb{R})}\left(\frac{v_{2}(t)}{v_{0,2}}\right)^{\frac{\lambda_{3}}{\lambda_{2}}} \exp \left(-\frac{\lambda_{3}-\lambda_{2}}{\lambda_{2}} \mu t\right) .
$$

In the remainder of this proof, $c$ denotes any constant that doesn't depend on $\varepsilon$ and that may change from line to line.

Thanks to assumption (2.4) on the spectral gap estimate for the eigenvalues $\lambda_{2}-\lambda_{3}$, we have

$$
\lambda_{3} / \lambda_{2}=1-\kappa(\varepsilon) \varepsilon<1 \text { with } \kappa(\varepsilon)>0, \forall \varepsilon>0 \text { and } \lim _{\varepsilon \rightarrow 0} \kappa(\varepsilon)>0 .
$$

Then for any $t \geq t_{k_{0}}^{\varepsilon}=\frac{k_{0}}{\varepsilon} \ln \left(\frac{1}{\varepsilon}\right)$ with $k_{0}>0$ and any $\varepsilon$ small enough, one has

$$
\left|R_{3}(t)\right| \leq c \varepsilon^{c k_{0}}\left(\frac{v_{2}(t)}{v_{0,2}}\right)^{1-\kappa(\varepsilon) \varepsilon} .
$$

Thanks to the previous lemma for all $\varepsilon$ small enough we have $1 \geq v_{0,2}^{\varepsilon} \geq c \varepsilon^{\frac{N}{2}}$, and

$$
\left(v_{0,2}^{\varepsilon}\right)^{-1+\kappa(\varepsilon) \varepsilon} \leq c \varepsilon^{-\frac{N}{2}},
$$

we choose $k_{0}$ such that $k=c k_{0}-N / 2>N / 2$, then

$$
\left|R_{3}(t)\right| \leq c \varepsilon^{k}\left(v_{2}(t)\right)^{1-\kappa(\varepsilon) \varepsilon}, \forall t \geq t_{k_{0}}^{\varepsilon} .
$$

Next using Young's inequality, for $\eta>0$ and $p, q \geq 1$ with $1 / p+1 / q=1$, we readily find that

$$
c \varepsilon^{k} v_{2}^{1-\kappa(\varepsilon) \varepsilon} \leq c^{p} \frac{\eta^{p}}{p} \varepsilon^{p k}+\frac{1}{q \eta^{q}} v_{2}^{q(1-\kappa(\varepsilon) \varepsilon)},
$$

so we choose the following values of $p, q$ and $\eta$

$$
q=\frac{1}{1-\kappa(\varepsilon) \varepsilon}, p=\frac{1}{\kappa(\varepsilon) \varepsilon}, \eta=\left(\frac{2}{\lambda_{2} \beta_{2}^{\varepsilon}}(1-\kappa(\varepsilon) \varepsilon)\right)^{1-\kappa(\varepsilon) \varepsilon},
$$

in order to have the following equality for the second term

$$
\frac{1}{q \eta^{q}} v_{2}^{q(1-\kappa(\varepsilon) \varepsilon)}=\frac{1}{2} \lambda_{2} \beta_{2}^{\varepsilon} v_{2}
$$


For the first term we obtain

$$
c^{p} \frac{\eta^{p}}{p} \varepsilon^{p k}=c^{p} \kappa(\varepsilon) \varepsilon\left(\frac{2}{\lambda_{2} \beta_{2}^{\varepsilon}}(1-\kappa(\varepsilon) \varepsilon)\right)^{\frac{1-\kappa(\varepsilon) \varepsilon}{\kappa(\varepsilon) \varepsilon}} \varepsilon^{\frac{k}{\kappa(\varepsilon) \varepsilon}} .
$$

Notice that

$$
(1-\kappa(\varepsilon) \varepsilon)^{\frac{1-\kappa(\varepsilon) \varepsilon}{\kappa(\varepsilon) \varepsilon}} \rightarrow e^{-1} \text { as } \varepsilon \rightarrow 0,
$$

and thanks to (4.23) we have $\beta_{2}^{\varepsilon} \geq c \varepsilon^{\frac{N}{2}}$ and $\beta_{2}^{\varepsilon}=o(1)$, hence for $\varepsilon$ small enough

$$
\left(\frac{2}{\lambda_{2} \beta_{2}^{\varepsilon}}\right)^{\frac{1-\kappa(\varepsilon) \varepsilon}{\kappa(\varepsilon) \varepsilon}} \leq\left(\frac{c}{\varepsilon^{N / 2}}\right)^{\frac{1}{\kappa(\varepsilon) \varepsilon}}
$$

so that

$$
c^{p} \frac{\eta^{p}}{p} \varepsilon^{p k} \leq c \varepsilon \exp \left(\frac{1}{\kappa(\varepsilon) \varepsilon}(\ln c+(k-N / 2) \ln \varepsilon)\right) .
$$

Since we chose $k>N / 2$, then $\lim _{\varepsilon \rightarrow 0} \frac{1}{\kappa(\varepsilon) \varepsilon}(\ln c+(k-N / 2) \ln \varepsilon)=-\infty$. Therefore for $\varepsilon$ small enough

$$
c^{p} \frac{\eta^{p}}{p} \varepsilon^{p k} \leq c \varepsilon,
$$

so that finally we have, for any $\varepsilon$ small enough and $t \geq t_{k_{0}}^{\varepsilon}$,

$$
\left|R_{3}(t)\right| \leq c \varepsilon+\frac{1}{2} \lambda_{2} \beta_{2}^{\varepsilon} v_{2} .
$$

Reporting this estimate in the first equation of (2.9) we retrieve that

$$
\forall t \geq t_{k_{0}}^{\varepsilon}, U^{\prime}(t) \leq \Lambda-U(t)-\lambda_{1} \beta_{1}^{\varepsilon} v_{1}(t) U(t)-\frac{1}{2} \lambda_{2} \beta_{2}^{\varepsilon} v_{2}(t) U(t)+c \varepsilon U(t),
$$

and the second equation of (2.9) yields

$$
\left(\beta_{1}^{\varepsilon} v_{1}\right)^{\prime}=\beta_{1}^{\varepsilon} v_{1}\left(\lambda_{1} U-\mu\right), \text { and }\left(\frac{1}{2} \beta_{2}^{\varepsilon} v_{2}\right)^{\prime}=\frac{1}{2} \beta_{2}^{\varepsilon} v_{2}\left(\lambda_{2} U-\mu\right) .
$$

Let $Q=U+\beta_{1}^{\varepsilon} v_{1}+\frac{1}{2} \beta_{2}^{\varepsilon} v_{2}$, assuming that $\varepsilon$ is small enough to ensure that $\min (\mu, 1-c \varepsilon) \geq m>0$ with $m$ independent of $\varepsilon$, then adding (4.25) and (4.26) yields

$$
Q^{\prime}(t) \leq \Lambda-m Q(t) \text { for all } t \geq t_{k_{0}}^{\varepsilon}
$$

so that

$$
\forall t \geq t_{k_{0}}^{\varepsilon}, Q(t) \leq Q\left(t_{k_{0}}^{\varepsilon}\right) e^{-m\left(t-t_{k_{0}}^{\varepsilon}\right)}+\Lambda
$$

Now to crudely estimate $Q\left(t_{k_{0}}^{\varepsilon}\right)$ let us recall that $\forall t \geq 0, U(t) \leq \max \left(\Lambda, U_{0}\right)$, hence from the second equation of (2.9) there exists a constant $c>0$ such that for all $n \in \mathbb{N}$ we have for any $t \geq 0$,

$$
\left|v_{n}(t)\right| \leq\left|v_{0, n}\right| e^{c t}
$$


so that as $\left|\beta_{i}^{\varepsilon}\right|=o(1)$ is bounded we finally have $Q(t) \leq c e^{c t}$ for all $t \geq 0$, which implies $Q\left(t_{k_{0}}^{\varepsilon}\right) \leq c e^{c t t_{k_{0}}^{\varepsilon}}$. Then, coming back to (4.27) we find

$$
\forall t \geq t_{k_{0}}^{\varepsilon}, Q(t) \leq c e^{c t_{k_{0}}^{\varepsilon}} e^{-m\left(t-t_{k_{0}}^{\varepsilon}\right)}+\Lambda,
$$

Let $t_{k_{1}}^{\varepsilon}=\frac{k_{1}}{\varepsilon} \ln \frac{1}{\varepsilon}$ with $k_{1}>k_{0}$ be given. Then

$$
\forall t \geq t_{k_{1}}^{\varepsilon}, Q(t) \leq c e^{c t_{k_{0}}^{\varepsilon}} e^{-m\left(t_{k_{1}}^{\varepsilon}-t_{k_{0}}^{\varepsilon}\right)}+\Lambda=c \varepsilon^{\frac{m k_{1}-(m+c) k_{0}}{\varepsilon}}+\Lambda,
$$

hence finally for $k_{1}$ large enough and $\forall t \geq t_{k_{1}}^{\varepsilon}, Q(t)=U(t)+\beta_{1}^{\varepsilon} v_{1}(t)+\frac{1}{2} \beta_{2}^{\varepsilon} v_{2}(t)$ is bounded uniformly with respect to $\varepsilon$.

Finally we prove the following estimates for $v_{1}$ and $R_{3}=\sum_{n=3}^{+\infty} \lambda_{n} \beta_{n} v_{n}$.

Lemma 4.5 Let $k_{1}$ be defined in the previous lemma. The following estimates hold true for $\varepsilon$ small enough

$$
\begin{gathered}
\forall t \in\left[t_{k_{1}}^{\varepsilon}, G(\varepsilon)^{-1}\right], \quad v_{1}(t) \leq c \varepsilon^{-N} C(\rho, \varepsilon)=o\left(\varepsilon^{\infty}\right), \\
\forall t \geq t_{k_{1}}^{\varepsilon}, \quad\left|R_{3}(t)\right| \leq c \varepsilon^{-N} \exp (-c \varepsilon t),
\end{gathered}
$$

wherein $c$ is a positive constant independent of $\varepsilon \ll 1$.

Proof. Using Lemma 3.1 we have the following identity

$$
\forall t \geq 0, \quad v_{1}(t)=v_{0,1}\left(\frac{v_{2}(t)}{v_{0,2}}\right)^{\lambda_{2} / \lambda_{1}} e^{\frac{\lambda_{1}-\lambda_{2}}{\lambda_{1}} \mu t} .
$$

Using estimate (4.22) we have, for $\varepsilon \ll 1$,

$$
\left(v_{0,2}\right)^{-\frac{\lambda_{2}}{\lambda_{1}}} \leq c \varepsilon^{-\frac{N}{2}}
$$

Hence due to Assumption 2.4 on the spectral gap and (4.21), there exists $\kappa \in \mathbb{R}$ independent of $\varepsilon$ such that

$$
\forall t \geq 0, \quad v_{1}(t) \leq c \varepsilon^{-N / 2} C(\rho, \varepsilon)\left(v_{2}(t)\right)^{\lambda_{2} / \lambda_{1}} e^{c G(\varepsilon) t} .
$$

Now using Lemma 4.4 and estimate (4.23) readily implies (4.28).

Next, coming back to (4.24), that is

$$
\forall t \geq 0,\left|R_{3}(t)\right| \leq\left|\lambda_{3}\right|\|\beta\|_{L^{2}(\mathbb{R})}\left\|v_{0}\right\|_{L^{2}(\mathbb{R})}\left(\frac{v_{2}(t)}{v_{0,2}}\right)^{\frac{\lambda_{3}}{\lambda_{2}}} e^{-\frac{\lambda_{3}-\lambda_{2}}{\lambda_{2}} \mu t},
$$

as above we use estimates (4.22) for $v_{0,2}$, Assumption 2.4 (ii) on the second spectral gap and Lemma 4.4 combined with (4.23) to retrieve (4.29) for $\varepsilon$ small enough. 


\subsection{Proof of Theorem 2.6}

To achieve this, we use the estimates of the previous section combined with arguments featuring some degree of similarity with the ones given for the proof of Theorem 2.2, but taking into account the dependence on $\varepsilon$ of the solution and of the parameters.

Thus we start with a lemma ensuring a kind of weak persistence property in some sense for the solution $\left(U^{\varepsilon}, v_{2}^{\varepsilon}\right)$ of $(2.9)$ on an interval of the form $\left[t_{k}^{\varepsilon}, t_{\ell}^{\varepsilon}\right]$ uniformly with respect to $\varepsilon$. Here we use for each real number $\ell$ the notation

$$
t_{\ell}^{\varepsilon}=\frac{\ell}{\varepsilon} \ln \frac{1}{\varepsilon} .
$$

And the following lemma holds true

Lemma 4.6 Let us recall that

$$
\mathcal{R}_{0}^{\varepsilon}=\frac{\lambda_{1}^{\varepsilon} \Lambda}{\mu}, \quad \lambda_{0}:=\|\Theta\|_{\infty}^{2}=\lim _{\varepsilon \rightarrow 0} \lambda_{1}^{\varepsilon}, \quad \mathcal{R}_{0}^{0}=\lim _{\varepsilon \rightarrow 0} \mathcal{R}_{0}^{\varepsilon}=\frac{\lambda_{0} \Lambda}{\mu},
$$

and $\operatorname{set}\left(U_{2}^{*}, V_{2}^{*}\right)=\frac{1}{\lambda_{0}}\left(\mu, \mathcal{R}_{0}^{0}-1\right)$.

Let $\alpha \in\left(0, V_{2}^{*}\right)$ be given. Then there exist positive constants $k_{2}>k_{1}$ and $\widetilde{k}_{\alpha}>1$ such that the following property holds for the product $\beta_{2}^{\varepsilon} v_{2}^{\varepsilon}$ : for each $k>k_{2}$ and each $\ell \geq \widetilde{k}_{\alpha} k$, there exists $\varepsilon_{0}>0$ small enough such that for any $\varepsilon \in\left(0, \varepsilon_{0}\right)$ one has

$$
t_{\ell}^{\varepsilon}<G(\varepsilon)^{-1} \text { and } \sup _{t \in\left[t_{k}^{\varepsilon}, t_{\ell}^{\varepsilon}\right]} \beta_{2}^{\varepsilon} v_{2}^{\varepsilon}(t) \geq \alpha,
$$

where $k_{1}$ is the constant defined in Lemma 4.4 above.

Proof. Set $P^{\varepsilon}(t):=-\lambda_{1}^{\varepsilon} \beta_{1}^{\varepsilon} v_{1}^{\varepsilon}(t)-R_{3}^{\varepsilon}(t)$. Thanks to Lemma 4.5, there exist two constants $k_{2}>k_{1}$ and $c_{1}>0$ independent of $\varepsilon$ such that for $\varepsilon$ small enough

$$
\forall t \in\left[t_{k_{2}}^{\varepsilon}, G(\varepsilon)^{-1}\right], \quad\left|P^{\varepsilon}(t)\right| \leq c \varepsilon^{c_{1}} .
$$

Next recalling that $\mathcal{R}_{0}^{0}=\frac{\lambda_{0} \Lambda}{\mu}>1$ and $\alpha \in\left(0, V_{2}^{*}\right)$, let us fix $k_{\alpha}>0$ such that

$$
\mu\left(\frac{\mathcal{R}_{0}^{0}}{1+\lambda_{0} \alpha}-1\right)>k_{\alpha}>0
$$

Setting $\widehat{k}_{\alpha}:=1+\frac{\mu}{k_{\alpha}}$, we complete the proof of our lemma by showing that for each $k>k_{2}$ and each $\ell \geq \widehat{k}_{\alpha} k$, there exists $\varepsilon_{0}>0$ small enough such that for any $\varepsilon \in\left(0, \varepsilon_{0}\right)$ one has

$$
t_{\ell}^{\varepsilon}<G(\varepsilon)^{-1} \text { and } \sup _{t \in\left[t_{k}^{\varepsilon}, t_{\ell}^{\varepsilon}\right]} \beta_{2}^{\varepsilon} v_{2}^{\varepsilon}(t) \geq \alpha .
$$

Recalling the assumption on the spectral gaps, $G(\varepsilon)|\ln \varepsilon| \ll \varepsilon$ as $\varepsilon \rightarrow 0$, and in order to prove this statement we argue by contradiction. To that end, we 
assume that there exist $k>k_{2}, \ell>\widetilde{k}_{\alpha} k$ and a sequence of positive numbers $\left\{\varepsilon_{n}\right\}_{n \geq 0}$ tending to zero such that for all $n \geq 0$

$$
t_{\ell}^{\varepsilon_{n}}<G\left(\varepsilon_{n}\right)^{-1} \text { and } \beta_{2}^{\varepsilon_{n}} v_{2}^{\varepsilon_{n}}(t)<\alpha, \forall t \in\left[t_{k}^{\varepsilon_{n}}, t_{\ell}^{\varepsilon_{n}}\right] .
$$

In this proof we shall set $v_{n}=\beta_{2}^{\varepsilon_{n}} v_{2}^{\varepsilon_{n}}, \lambda_{n}=\lambda_{2}^{\varepsilon_{n}}$ and $\left(U_{n}, v_{n}\right)=\left(U^{\varepsilon_{n}}, v^{\varepsilon_{n}}\right)$ for the sake of simplicity. Then $\left(U_{n}, v_{n}\right)$ satisfies the non autonomous system

$$
\left\{\begin{array}{l}
U_{n}^{\prime}(t)=\Lambda-U_{n}(t)-\lambda_{n} v_{n}(t) U_{n}(t)+P_{n}(t) U_{n}(t), \\
v_{n}^{\prime}(t)=v_{n}(t)\left(\lambda_{n} U_{n}(t)-\mu\right),
\end{array}\right.
$$

wherein we have set $P_{n}(t)=P^{\varepsilon_{n}}(t)$.

In the remainder of this proof, let $c$ denote any constant independent of $n$. First due to the choice of $k_{2}>k_{1}$ one has (see (4.30))

$$
\left|P_{n}(t)\right| \leq c \varepsilon_{n}^{c_{1}}, \forall t \geq t_{k}^{\varepsilon_{n}}, \forall n \geq 0 .
$$

Next from the first equation of (4.32) and (4.33) we have for all $n \geq 0$ and $t \in\left[t_{k}^{\varepsilon_{n}}, t_{\ell}^{\varepsilon_{n}}\right]$,

$$
U_{n}^{\prime}(t) \geq \Lambda-c_{\alpha}\left(\varepsilon_{n}\right) U_{n}(t)
$$

with

$$
c_{\alpha}\left(\varepsilon_{n}\right)=1+\alpha \lambda_{n}+c \varepsilon_{n}{ }^{c_{1}}>0 .
$$

Hence for any $n \geq 0$ one has

$$
\forall t \in\left[t_{k}^{\varepsilon_{n}}, t_{\ell}^{\varepsilon_{n}}\right], U_{n}(t) \geq \frac{\Lambda}{c_{\alpha}\left(\varepsilon_{n}\right)}+\left(U\left(t_{k}^{\varepsilon_{n}}\right)-\frac{\Lambda}{c_{\alpha}\left(\varepsilon_{n}\right)}\right) e^{-c_{\alpha}\left(\varepsilon_{n}\right)\left(t-t_{k}^{\varepsilon_{n}}\right)} .
$$

Replacing this estimate in the right-hand side of the second equation of (4.32) and integrating we find for any $t \in\left[t_{k}^{\varepsilon_{n}}, t_{\ell}^{\varepsilon_{n}}\right]$ :

$$
v_{n}(t) \geq v_{n}\left(t_{k}^{\varepsilon_{n}}\right) e^{\left(\frac{\lambda_{n} \Lambda}{c_{\alpha}\left(\varepsilon_{n}\right)}-\mu\right)\left(t-t_{k}^{\varepsilon_{n}}\right)} \times e^{\lambda\left(U_{n}\left(t_{k}^{\varepsilon_{n}}\right)-\frac{\Lambda}{c_{\alpha}\left(\varepsilon_{n}\right)}\right) \int_{t_{k}^{\varepsilon_{n}}}^{t} e^{-c_{\alpha}\left(\varepsilon_{n}\right)\left(s-t_{k}^{\varepsilon_{n}}\right)} \mathrm{d} s} .
$$

Let us estimate the factors in right hand-side of (4.34). From the second equation of (4.32), $\forall t \geq 0, v_{n}^{\prime}(t) \geq-\mu v_{n}(t)$, hence

$$
v_{n}\left(t_{k}^{\varepsilon_{n}}\right) \geq \beta_{2}^{\varepsilon_{n}} v_{2}^{\varepsilon_{n}}(0) \exp \left(-\mu t_{k}^{\varepsilon_{n}}\right)
$$

and, using estimates (4.22) and (4.23) on $v_{2}^{\varepsilon}(0)$ and $\beta_{2}^{\varepsilon}$ we have $\beta_{2}^{\varepsilon_{n}} v_{2}^{\varepsilon_{n}}(0) \geq c \varepsilon_{n}^{N}$, and therefore

$$
v_{n}\left(t_{k}^{\varepsilon_{n}}\right) \geq c \exp \left(\left(N+\mu k / \varepsilon_{n}\right) \ln \varepsilon_{n}\right) .
$$

To estimate the second factor in (4.34), note that, as $n \rightarrow \infty$,

$$
\left(\frac{\lambda_{n} \Lambda}{c_{\alpha}\left(\varepsilon_{n}\right)}-\mu\right) \rightarrow \mu\left(\frac{\mathcal{R}_{0}^{0}}{1+\lambda_{0} \alpha}-1\right)>k_{\alpha}>0 .
$$


So, for any $n$ large enough one has

$$
\frac{\lambda_{n} \Lambda}{c_{\alpha}\left(\varepsilon_{n}\right)}-\mu \geq k_{\alpha}>0
$$

so that

$$
e^{\left(\frac{\lambda_{n} \Lambda}{c_{\alpha}\left(\varepsilon_{n}\right)}-\mu\right)\left(t_{\ell}^{\varepsilon n}-t_{k}^{\varepsilon_{n}}\right)} \geq e^{\frac{k_{\alpha}(\ell-k)}{\varepsilon_{n}} \ln \frac{1}{\varepsilon_{n}}} .
$$

For the third factor in (4.34) one has

$$
\lambda_{n}\left(U_{n}\left(t_{k}^{\varepsilon_{n}}\right)-\frac{\Lambda}{c_{\alpha}\left(\varepsilon_{n}\right)}\right) \int_{t_{k}^{\varepsilon_{n}}}^{t} e^{-c_{\alpha}\left(\varepsilon_{n}\right)\left(s-t_{k}^{\varepsilon_{n}}\right)} \mathrm{d} s \geq-\frac{\lambda_{n}}{c_{\alpha}\left(\varepsilon_{n}\right)}\left|U_{n}\left(t_{k}^{\varepsilon_{n}}\right)-\frac{\Lambda}{c_{\alpha}\left(\varepsilon_{n}\right)}\right|,
$$

and $U_{n}(t)$ being bounded uniformly with respect to $\varepsilon_{n}$ and non negative, the third factor is greater than some negative constant.

Finally we recover from (4.34) that

$$
v_{n}\left(t_{\ell}^{\varepsilon_{n}}\right) \geq c e^{\left(\frac{k_{\alpha}(\ell-k)-\mu k}{\varepsilon_{n}}-N\right) \ln \frac{1}{\varepsilon_{n}}},
$$

so that, since $\ell>\widetilde{k}_{\alpha} k$, one has $k_{\alpha}(\ell-k)-\mu k>0$. And for any $n$ large enough we obtain $v_{n}\left(t_{\ell}^{\varepsilon_{n}}\right) \geq \alpha$, which contradicts (4.31). This concludes the proof of this lemma.

Next we are able to prove a lemma ensuring a kind of strong persistence property for $v_{2}^{\varepsilon}$ on some interval of the form $\left[t_{k}^{\varepsilon}, G(\varepsilon)^{-1}\right]$ uniformly with respect to $\varepsilon$. The proof of this lemma is rather similar to the proof of Lemma 3.5.

Lemma 4.7 Let $\alpha \in\left(0, V_{2}^{*}\right)$ be given. Let $k>k_{2}$ and $\ell>\widetilde{k}_{\alpha} k$ given. For each $\varepsilon>0$ small enough, let $k_{\varepsilon} \in[k, \ell]$ be given and provided by Lemma 4.6 such that $\beta_{2}^{\varepsilon} v_{2}^{\varepsilon}\left(t_{k_{\varepsilon}}^{\varepsilon}\right) \geq \alpha$. Then there exists $\eta>0$ such that for all $\varepsilon$ small enough

$$
\beta_{2}^{\varepsilon} v_{2}^{\varepsilon}(t) \geq \eta \text { for any } t \in\left[t_{k_{\varepsilon}}^{\varepsilon}, G(\varepsilon)^{-1}\right] .
$$

Remark 4.8 In particular, since $k_{\varepsilon} \leq \ell$ one also has for all $\varepsilon$ small enough

$$
\beta_{2}^{\varepsilon} v_{2}^{\varepsilon}(t) \geq \eta, \forall t \in\left[t_{\ell}^{\varepsilon}, G(\varepsilon)^{-1}\right] .
$$

Proof. To prove this lemma we argue by contradiction and we assume that exists a sequence of positive numbers $\left(\varepsilon_{n}\right)_{n \geq 0}$ that tends to zero and a sequence $\left(T_{n}\right)_{n \geq 0}$ with

$$
T_{n} \in\left[t_{k_{\varepsilon_{n}}}^{\varepsilon_{n}}, G\left(\varepsilon_{n}\right)^{-1}\right] \text { such that } \lim _{n \rightarrow+\infty} \beta_{2}^{\varepsilon_{n}} v_{2}^{\varepsilon_{n}}\left(T_{n}\right)=0 .
$$

As in the proof of the previous lemma we set $U_{n}(t)=U^{\varepsilon_{n}}(t), v_{n}(t)=$ $\beta_{2}^{\varepsilon_{n}} v_{2}^{\varepsilon_{n}}(t)$ and we define $X_{n}=\left(U_{n}, v_{n}\right)$ a solution of (4.32). We also set $t_{n}=$ $t_{k_{\varepsilon_{n}}}^{\varepsilon_{n}}$.

Now as $v_{n}\left(T_{n}\right) \rightarrow 0$ as $n \rightarrow \infty$ and $v_{n}\left(t_{n}\right) \geq \alpha>0$, there exists a sequence $\left(h_{n}\right)$ defined for $n$ large enough by

$$
h_{n}=\inf \left\{h \in\left(0, T_{n}-t_{n}\right), v\left(T_{n}-h\right)=\frac{\alpha}{2}\right\} .
$$


In other words one has $v_{n}\left(T_{n}-h_{n}\right)=\frac{\alpha}{2}, v_{n}(t) \leq \frac{\alpha}{2}$ on $\left[T_{n}-h_{n}, T_{n}\right]$ and $v_{n}\left(T_{n}\right) \rightarrow 0$ as $n \rightarrow \infty$. Next since $v_{n}\left(t_{n}+\cdot\right)$ is uniformly bounded on $[0, \infty)$ (see Lemma 4.4) as well as its derivative, and since it satisfies the second equation in (4.32), it readily follows from Arzelà-Ascoli compactness theorem that $h_{n} \rightarrow+\infty$ as $n \rightarrow \infty$ (see the similar proof of Lemma 3.5 for more details). Moreover, one may assume, possibly along a subsequence, that the sequence of functions $\left(U_{n}, v_{n}\right)\left(T_{n}-h_{n}+t\right) \rightarrow\left(U_{\infty}, v_{\infty}\right)(t)$ locally uniformly for $t \geq 0$ wherein $\left(U_{\infty}, v_{\infty}\right)$ becomes a bounded non-negative orbit of the autonomous system corresponding to system (4.32) with $P_{n} \equiv 0$ and $\lambda_{n}=\lambda_{0}$, i.e.

$$
\left\{\begin{array}{l}
U_{\infty}^{\prime}(t)=\Lambda-U_{\infty}(t)-\lambda_{0} v_{\infty}(t) U_{\infty}(t), \\
v_{\infty}^{\prime}(t)=v_{\infty}(t)\left(\lambda_{0} U_{\infty}(t)-\mu\right),
\end{array}\right.
$$

that furthermore satisfies $v_{\infty}(0)=\frac{\alpha}{2}$ and $v_{\infty}(t) \leq \frac{\alpha}{2}, \forall t \geq 0$. However such a solution doesn't exist by applying Lemma 3.3 (iii) and recalling that $\mathcal{R}_{0}^{0}>1$ and $\alpha<V_{2}^{*}$. This contradiction completes the proof of the lemma.

Before going to the proof of Theorem 2.6 let us prove the following proposition that roughly shows that the function $\beta_{2}^{\varepsilon} v_{2}^{\varepsilon}(t)$ remains in a small neighbourhood of $V_{2}^{*}=\frac{\mathcal{R}_{0}^{0}-1}{\lambda_{0}}$ on some interval of the form $\left[t_{k}^{\varepsilon}, G(\varepsilon)^{-1}\right]$ for any $\varepsilon \ll 1$.

Proposition 4.9 Assume $\mathcal{R}_{0}^{0}>1$. There exists $k>1$ large enough such that for any $\delta>0$ there exists $\varepsilon_{0}>0$ small enough such that for all $\varepsilon \in\left(0, \varepsilon_{0}\right)$ one has

$$
\left|U^{\varepsilon}(t)-U_{2}^{\varepsilon}\right|+\left|\beta_{2}^{\varepsilon} v_{2}^{\varepsilon}(t)-\beta_{2}^{\varepsilon} V_{2}^{\varepsilon}\right| \leq \delta, \forall t \in\left[t_{k}^{\varepsilon}, G(\varepsilon)^{-1}-t_{1}^{\varepsilon}\right] .
$$

Proof. Fix $\alpha \in\left(0, V_{2}^{*}\right)$. Let $k>\widetilde{k}_{\alpha} k_{2}$ be given. Hence Lemma 4.7 applies and ensures that

$$
\beta_{2}^{\varepsilon} v_{2}^{\varepsilon}(t) \geq \eta \text { for } t \in\left[t_{k}^{\varepsilon}, G(\varepsilon)^{-1}\right]
$$

for some positive constant $\eta$ independent of $\varepsilon$ and for any $\varepsilon \ll 1$ small enough.

To prove Proposition 4.9 we fix $\widehat{k}>k$ and we again argue by contradiction by assuming that there exist $\delta_{0}$ and a sequence $\left(\varepsilon_{n}\right)$ that tends to 0 such that for all $t_{k}^{\varepsilon_{n}}<\frac{1}{G\left(\varepsilon_{n}\right)}$ we have

$$
\sup _{t \in\left[t_{\hat{k}}^{\varepsilon_{n}}, \frac{1}{G\left(\varepsilon_{n}\right)}-t_{1}^{\varepsilon_{n}}\right]}\left(\left|U^{\varepsilon_{n}}(t)-U_{2}^{\varepsilon_{n}}\right|+\left|\beta_{2}^{\varepsilon_{n}} v^{\varepsilon_{n}}(t)-\beta_{2}^{\varepsilon} V_{2}^{\varepsilon_{n}}\right|\right)>\delta_{0} .
$$

Hence there is a sequence $\left(t_{n}\right)$ such that $t_{n} \in\left[t_{\widehat{k}}^{\varepsilon_{n}}, \frac{1}{G\left(\varepsilon_{n}\right)}-t_{1}^{\varepsilon_{n}}\right]$ and

$$
\left|U^{\varepsilon_{n}}\left(t_{n}\right)-U_{2}^{\varepsilon_{n}}\right|+\left|\beta_{2}^{\varepsilon_{n}} v^{\varepsilon_{n}}\left(t_{n}\right)-\beta_{2}^{\varepsilon} V_{2}^{\varepsilon_{n}}\right|>\delta_{0} .
$$

As for the proof of the previous lemma by considering

$$
Y^{\varepsilon_{n}}(t):=\left(U^{\varepsilon_{n}}\left(t+t_{n}\right), \beta_{2}^{\varepsilon_{n}} v^{\varepsilon_{n}}\left(t+t_{n}\right)\right), \text { for }\left(t+t_{n}\right) \in\left[t_{k}^{\varepsilon_{n}}, G\left(\varepsilon_{n}\right)^{-1}\right] .
$$

Since $t_{k}^{\varepsilon_{n}}-t_{n} \leq t_{k}^{\varepsilon_{n}}-t_{\widehat{k}}^{\varepsilon_{n}} \rightarrow-\infty$ and $G\left(\varepsilon_{n}\right)^{-1}-t_{n} \geq t_{1}^{\varepsilon_{n}} \rightarrow+\infty$ as $n \rightarrow \infty$, possibly along a sub-sequence, it converges locally uniformly for $t \in \mathbb{R}$ toward 
some limit function $\left(U_{\infty}, V_{\infty}\right)(t)$ as $n$ tends to infinity. This limit function becomes a complete orbit of (4.35) that satisfies $\left(U_{\infty}, V_{\infty}\right)(0) \neq\left(U^{*}, V^{*}\right)$ and $V_{\infty}(t) \geq \eta>0, \forall t \in \mathbb{R}$. This contradicts Lemma 3.3 (ii) and concludes the proof of Proposition 4.9.

We complete this section by the proof of Theorem 2.6.

Proof of Theorem 2.6.

For the sake of simplicity we set $v^{\varepsilon}=\beta_{2}^{\varepsilon} v_{2}^{\varepsilon}, \lambda^{\varepsilon}=\lambda_{2}^{\varepsilon}$. Then $\left(U^{\varepsilon}, v^{\varepsilon}\right)$ satisfies the non autonomous system

$$
\left\{\begin{array}{l}
\frac{\mathrm{d} U^{\varepsilon}}{\mathrm{d} t}(t)=\Lambda-U^{\varepsilon}(t)-\lambda^{\varepsilon} v^{\varepsilon}(t) U^{\varepsilon}(t)+P^{\varepsilon}(t) U^{\varepsilon}(t), \\
\frac{\mathrm{d} v^{\varepsilon}}{\mathrm{d} t}(t)=v^{\varepsilon}(t)\left(\lambda^{\varepsilon} U^{\varepsilon}(t)-\mu\right),
\end{array}\right.
$$

where again $P^{\varepsilon}(t):=-\lambda_{1}^{\varepsilon} \beta_{1}^{\varepsilon} v_{1}^{\varepsilon}(t)-R_{3}^{\varepsilon}(t)$.

We linearise this system around $\left(U_{2}^{\varepsilon}, V_{2}^{\varepsilon}\right)$ defined in (2.8). So we set $V^{\varepsilon}(t)=$ $U^{\varepsilon}(t)-U_{2}^{\varepsilon}, w^{\varepsilon}(t)=v^{\varepsilon}(t)-\beta_{2}^{\varepsilon} V_{2}^{\varepsilon}$ and $Y^{\varepsilon}(t)=\left(V^{\varepsilon}(t), w^{\varepsilon}(t)\right)$. Then we readily compute that

$$
\frac{\mathrm{d} Y^{\varepsilon}}{\mathrm{d} t}=A_{\varepsilon} Y^{\varepsilon}+F_{\varepsilon}\left(Y^{\varepsilon}\right)+G_{\varepsilon}\left(t, Y^{\varepsilon}\right)
$$

where $A_{\varepsilon}, F_{\varepsilon}$ and $G_{\varepsilon}$ are respectively defined by

$A_{\varepsilon}=\left(\begin{array}{cc}-\mathcal{R}_{0,2}^{\varepsilon} & -\mu \\ \mathcal{R}_{0,2}^{\varepsilon}-1 & 0\end{array}\right), F_{\varepsilon}\left(Y^{\varepsilon}\right)=\left(\begin{array}{c}-\lambda_{\varepsilon} V^{\varepsilon} w^{\varepsilon} \\ \lambda_{\varepsilon} V^{\varepsilon} w^{\varepsilon}\end{array}\right), G_{\varepsilon}\left(t, Y^{\varepsilon}\right)=\left(\begin{array}{c}P^{\varepsilon}(t)\left(U_{2}^{\varepsilon}+V^{\varepsilon}\right) \\ 0\end{array}\right)$

Let us remark that $\operatorname{tr}\left(A_{\varepsilon}\right)=-\mathcal{R}_{0,2}^{\varepsilon}$ and $\operatorname{det}\left(A_{\varepsilon}\right)=\mu\left(\mathcal{R}_{0,2}^{\varepsilon}-1\right)$. As $\lim _{\varepsilon \rightarrow 0} \mathcal{R}_{0,2}^{\varepsilon}=$ $\mathcal{R}_{0}^{0}>1$ we readily prove that the real parts of the eigenvalues of $A_{\varepsilon}$ are negative and uniformly bounded away from 0 for $\varepsilon$ small enough. Moreover as the coefficients of $A_{\varepsilon}$ are bounded, there are positive constants $\alpha$ and $M$ that don't depend on $\varepsilon$ such that, for all $X \in \mathbb{R}^{2}$ and $t \geq 0,\left\|e^{A t} X\right\| \leq M e^{-\alpha t}\|X\|$ where $\|\cdot\|$ is the $\ell^{1}$ norm on $\mathbb{R}^{2}$.

By definition of $F_{\varepsilon}$ and as $\lambda_{\varepsilon}$ is bounded there exists some constant $K$ independent of $\varepsilon$ such that

$$
\forall Y \in \mathbb{R}^{2},\left\|F_{\varepsilon}(Y)\right\| \leq K\|Y\|^{2}
$$

Let $\delta>0$ such that $\delta M K \leq \alpha / 2$. Due to Proposition 4.9 there exists $k>0$ such that

$$
\forall t \in\left[t_{k}^{\varepsilon}, G(\varepsilon)^{-1}-t_{1}^{\varepsilon}\right],\left\|Y^{\varepsilon}(t)\right\| \leq \delta .
$$

Again in the remainder of this proof $c$ will denote any positive constant independent of $\varepsilon$. Thanks to Lemma 4.5, for any $p>N / 2$ there exist some real number $k_{1}>0$ and a constant $c$ such that we have

$$
\forall t \geq t_{k_{1}}^{\varepsilon},\left|P^{\varepsilon}(t)\right| \leq c \varepsilon^{p}
$$


We set $k=\max \left\{k, k_{1}\right\}$ for simplicity's sake. Then Duhamel's formula writes

$$
Y^{\varepsilon}(t)=e^{A_{\varepsilon}\left(t-t_{k}^{\varepsilon}\right)} Y^{\varepsilon}\left(t_{k}^{\varepsilon}\right)+\int_{t_{k}^{\varepsilon}}^{t} e^{A_{\varepsilon}(t-s)}\left(F_{\varepsilon}\left(Y^{\varepsilon}(s)\right)+G_{\varepsilon}\left(s, Y^{\varepsilon}(s)\right)\right) \mathrm{d} s .
$$

Thanks to (4.36), (4.37), (4.38), and as $U^{\varepsilon}$ is bounded by a constant independent of $\varepsilon$, we have for all $t \in\left[t_{k}^{\varepsilon}, G(\varepsilon)^{-1}-t_{1}^{\varepsilon}\right]$

$$
\left\|Y^{\varepsilon}(t)\right\| \leq \delta M e^{-\alpha\left(t-t_{k}^{\varepsilon}\right)}+M \int_{t_{k}^{\varepsilon}}^{t} e^{-\alpha(t-s)}\left(\delta K\left\|Y^{\varepsilon}(s)\right\|+c \varepsilon^{p}\right) \mathrm{d} s,
$$

then, as $\delta M K \leq \alpha / 2$, we retrieve that for all $t \in\left[t_{k}^{\varepsilon}, G(\varepsilon)^{-1}-t_{1}^{\varepsilon}\right]$

$$
\left\|Y^{\varepsilon}(t)\right\| \leq \delta M e^{-\alpha\left(t-t_{k}^{\varepsilon}\right)}+c \varepsilon^{p}+\frac{\alpha}{2} \int_{t_{k}^{\varepsilon}}^{t} e^{-\alpha(t-s)}\left\|Y^{\varepsilon}(s)\right\| \mathrm{d} s .
$$

Next it follows from Grönwall inequality that for all $t \in\left[t_{k}^{\varepsilon}, G(\varepsilon)^{-1}-t_{1}^{\varepsilon}\right]$

$$
e^{\alpha t}\left\|Y^{\varepsilon}(t)\right\| \leq \delta M e^{\alpha t_{k}^{\varepsilon}}+c \varepsilon^{p} e^{\alpha t}+\frac{\alpha}{2} \int_{t_{k}^{\varepsilon}}^{t}\left(\delta M e^{\alpha t_{k}^{\varepsilon}}+c \varepsilon^{p} e^{\alpha s}\right) e^{\frac{\alpha}{2}(t-s)} \mathrm{d} s,
$$

and we have the following upper estimates

$$
\frac{\alpha}{2} \int_{t_{k}^{\varepsilon}}^{t} \delta M e^{\alpha t_{k}^{\varepsilon}} e^{\frac{\alpha}{2}(t-s)} \mathrm{d} s \leq \delta M e^{\frac{\alpha}{2}\left(t+t_{k}^{\varepsilon}\right)}, \frac{\alpha}{2} \int_{t_{k}^{\varepsilon}}^{t} c \varepsilon^{p} e^{\alpha s} e^{\frac{\alpha}{2}(t-s)} \mathrm{d} s \leq c \varepsilon^{p} e^{\frac{\alpha}{2}\left(t-t_{k}^{\varepsilon}\right)} .
$$

Finally we have proved that

$$
\left\|Y^{\varepsilon}(t)\right\| \leq \delta M e^{-\alpha\left(t-t_{k}^{\varepsilon}\right)}+c \varepsilon^{p}+\delta M e^{-\frac{\alpha}{2}\left(t-t_{k}^{\varepsilon}\right)}+c \varepsilon^{p} e^{-\frac{\alpha}{2}\left(t+t_{k}^{\varepsilon}\right)},
$$

therefore there exists $\ell>k$ such that for all $t \in\left[t_{\ell}^{\varepsilon}, G(\varepsilon)^{-1}-t_{1}^{\varepsilon}\right]$ and $\varepsilon$ small enough $\left\|Y^{\varepsilon}(t)\right\| \leq c \varepsilon^{p}$. Then coming back to the definition of $Y^{\varepsilon}$ and thanks to estimate (4.23) for $\beta_{2}$ we have for all $t \in\left[t_{\ell}^{\varepsilon}, G(\varepsilon)^{-1}-t_{1}^{\varepsilon}\right]$

$$
\left|U^{\varepsilon}(t)-U_{2}^{\varepsilon}\right| \leq c \varepsilon^{p},\left|v_{2}^{\varepsilon}(t)-V_{2}^{\varepsilon}\right| \leq c \varepsilon^{p}\left(\beta_{2}^{\varepsilon}\right)^{-1} \leq c \varepsilon^{p-N / 2} .
$$

Finally, as the eigenvalues $\lambda_{n}^{\varepsilon}$ are uniformly bounded, with a slight modification of the proof of Lemma 4.5 we readily establish that

$$
\forall t \in\left[t_{k_{1}}^{\varepsilon}, G(\varepsilon)^{-1}\right],\left|\beta_{1}^{\varepsilon} v_{1}^{\varepsilon}(t)\right| \leq c \varepsilon^{-N} C(\rho, \varepsilon)=o\left(\varepsilon^{\infty}\right),
$$

and

$$
\forall t \geq t_{k_{1}}^{\varepsilon},\left\|\sum_{n=3}^{+\infty} \beta_{n}^{\varepsilon} v_{n}^{\varepsilon}(t) \varphi_{n}(\cdot)\right\|_{L^{2}(\mathbb{R})} \leq c \varepsilon^{-N} \exp (-c \varepsilon t) .
$$

Hence let $q=p-N / 2>0$ and we have for all $\varepsilon$ small enough

$$
\forall t \in\left[t_{\ell}^{\varepsilon}, G(\varepsilon)^{-1}-t_{1}^{\varepsilon}\right],\left|U^{\varepsilon}(t)-U_{2}^{\varepsilon}\right|+\left\|v^{\varepsilon}(t, .)-V_{2}^{\varepsilon} \varphi_{2}^{\varepsilon}(.)\right\|_{L^{2}\left(\mathbb{R}^{N}\right)} \leq c \varepsilon^{q} .
$$

This concludes the proof of Theorem 2.6. 


\section{Acknowledgements}

R. D. D. received support from the CIVB (Conseil Interprofessionnel du Vin de Bordeaux) and from the EU in the framework of the Marie-Curie FP7 COFUND People Programme, through the award of an AgreenSkills/AgreenSkills+ fellowship under grant agreement Number FP7-609398.

\section{References}

[1] Alikakos N.D. and Fusco G. 1998. Slow dynamics for the Cahn-Hilliard equation in higher space dimensions: the motion of bubbles, Arch. Rational Mech. Anal. 141(1), pp. 1-61.

[2] Bonnefon O., Coville J. and Legendre G., 2017. Concentration phenomenon in some non-local equation, Discrete Cont Dyn-B, 22(3), pp. 763-781.

[3] Calsina À., Cuadrado S., Desvillettes L. and Raoul G., 2013. Asymptotics of steady states of a selection-mutation equation for small mutation rate. Proc. Math. Roy. Soc. Edinb., 143, pp. 1123-1146.

[4] Calsina, À., \& Cuadrado, S., 2005. Stationary solutions of a selection mutation model: The pure mutation case. Mathematical Models and Methods in Applied Sciences, 15(07), 1091-1117.

[5] Carr J. and Pego R. L., 1989. Metastable patterns in solutions of $\partial_{t} u=$ $\varepsilon^{2} u_{x x}-f(u)$, Comm. Pure Appl. Math. 42(5), pp. 523-576.

[6] Cuadrado S., 2009. Equilibria of a predator prey model of phenotype evolution, J. Math. Anal. Appl. 354, pp. 286-294.

[7] Day T. and Gandon S., 2007. Applying population-genetic models in theoretical evolutionary epidemiology, Ecology Letters, 10, pp. 876-888.

[8] Diekmann O., Jabin P.E., Mischler S. and Perthame B., 2005. The dynamics of adaptation: an illuminating example and a Hamilton-Jacobi approach. Theor. Popul. Biol., 67, pp. 257-271.

[9] Djidjou-Demasse R., Ducrot A. and Fabre F., 2017. Steady state concentration for a phenotypic structured problem modeling the evolutionary epidemiology of spore producing pathogens. Mathematical Models and Methods in Applied Sciences, 27(02), pp. 385-426.

[10] Ducrot A., 2016. Spatial propagation for a two component reactiondiffusion system arising in population dynamics, J. Differential Equations, 260, pp. 8316-8357.

[11] Fusco G. and Hale J. K., 1989. Slow-motion manifolds, dormant instability, and singular perturbations, J. Dynam. Differential Equations 1(1), pp. 7594 . 
[12] Geritz S.A., Metz J.A., Kisdi É. and Meszéna G., 1997. Dynamics of adaptation and evolutionary branching. Phys. Rev. Lett., 78(10), pp. 2024-2027.

[13] Iacono G.L., van den Bosch F. and Paveley N., 2012. The evolution of plant pathogens in response to host resistance: factors affecting the gain from deployment of qualitative and quantitative resistance. J. Theo. Biol., 304, pp. 152-163.

[14] Laforgue J.G.L. and O'Malley R.E. Jr., 1994. On the motion of viscous shocks and the super-sensitivity of their steady-state limits, Methods Appl. Anal. 1(4), pp. 465-487.

[15] Lannou C., 2012. Variation and selection of quantitative traits in plant pathogens. Annu. Rev. Phytopathol., 50, pp. 319-338.

[16] Mascia C. and Strani M., 2013. Metastability for nonlinear parabolic equations with application to scalar viscous conservation laws. SIAM Journal on Mathematical Analysis, 45(5), 3084-3113.

[17] Metz J.A.J., Geritz S.A.H., Meszéna G., Jacobs F.J.A. and van Heerwaarden J.S., 1996. Adaptive dynamics, a geometrical study of the consequences of nearly faithful reproduction. In: van Strien, S.J., Verduyn Lunel, S.M. (Eds.), Stochastic and spatial Structures of Dynamical Systems. NorthHolland, Amsterdam, pp. 183-231.

[18] van Nimwegen E. and Crutchfield J.P., 2000. Metastable evolutionary dynamics: Crossing fitness barriers or escaping via neutral paths? Bull. Math. Biol., 62, pp. 799-848.

[19] Pego R.L., 1989. Front migration in the nonlinear Cahn-Hilliard equation, Proc. Roy. Soc. London Ser. A 422 (1863), 26-278.

[20] Reyna L.G. and Ward M.J., 1995. On the exponentially slow motion of a viscous shock, Comm. Pure Appl. Math. 48(2), 79-120.

[21] Sun X. and Ward M.J., 1999. Metastability for a generalized Burgers equation with applications to propagating flame-fronts, European J. Appl. Math. 10(1), 27-53.

[22] Wilke C.O., Wang J.L., Ofria C., Lenski R.E. and Adami C., 2001. Evolution of digital organisms at high mutation rates leads to survival of the flattest. Nature, 412, pp. 331-333.

[23] Zhan J., Thrall P.H., Papaïx J., Xie L. and Burdon J.J., 2015. Playing on a pathogen's weakness: using evolution to guide sustainable plant disease control strategies. Annu. Rev. Phytopathol., 53, pp. 19-43. 